\title{
SiamL.
}

\section{Slow Passage Through a Pitchfork Bifurcation}

Author(s): G. J. M. Maree

Source: SIAM Journal on Applied Mathematics, Vol. 56, No. 3 (Jun., 1996), pp. 889-918

Published by: Society for Industrial and Applied Mathematics

Stable URL: http://www.jstor.org/stable/2102651

Accessed: 09/02/2011 11:21

Your use of the JSTOR archive indicates your acceptance of JSTOR's Terms and Conditions of Use, available at http://www.jstor.org/page/info/about/policies/terms.jsp. JSTOR's Terms and Conditions of Use provides, in part, that unless you have obtained prior permission, you may not download an entire issue of a journal or multiple copies of articles, and you may use content in the JSTOR archive only for your personal, non-commercial use.

Please contact the publisher regarding any further use of this work. Publisher contact information may be obtained at http://www.jstor.org/action/showPublisher?publisherCode=siam.

Each copy of any part of a JSTOR transmission must contain the same copyright notice that appears on the screen or printed page of such transmission.

JSTOR is a not-for-profit service that helps scholars, researchers, and students discover, use, and build upon a wide range of content in a trusted digital archive. We use information technology and tools to increase productivity and facilitate new forms of scholarship. For more information about JSTOR, please contact support@jstor.org. 


\title{
SLOW PASSAGE THROUGH A PITCHFORK BIFURCATION*
}

\author{
G. J. M. MARÉE ${ }^{\dagger}$
}

\begin{abstract}
This paper deals with a class of second-order differential equations with a slowly varying bifurcation parameter. The parameter slowly varies through a critical value corresponding to a transition from a stable equilibrium to one of the two stable branches of an intersecting parabolic curve. The local transition behaviour is described by the second Painleve transcendent. In this study we predict which branch will be followed after passage of the bifurcation point given the initial state. For that purpose, use is made of averaging methods and of asymptotic matching techniques connecting local solutions valid before, during, and after the transition.
\end{abstract}

Key words. slowly varying parameter, pitchfork bifurcation, nonlinear oscillator, averaging, matched asymptotic expansions, second Painlevé transcendent

AMS subject classifications. $34 \mathrm{C} 15,34 \mathrm{C} 23,24 \mathrm{C} 29,34 \mathrm{E} 05,34 \mathrm{E} 10$

1. Introduction. We consider a second-order nonlinear differential equation depending on a parameter $F$ :

$$
\frac{d^{2} x}{d t^{2}}+k \frac{d x}{d t}=G(x, F) .
$$

The damping is assumed to be fixed and small, $k=\kappa \varepsilon$, while the parameter $F$ slowly varies in time. We have chosen a damping of order $O(\varepsilon)$, because the effect of the damping is then of order $O(1)$ on a time-scale $O(1 / \varepsilon)$. In the literature the effect of the dissipation is also often $O(\varepsilon)$. The matched asymptotic results of this study can be extended to a damping of order $O\left(\varepsilon^{\alpha}\right), \alpha>1 / 3$. In this study we analyse a class of bifurcation problems represented by the prototype system

$$
\begin{aligned}
\frac{d^{2} x}{d t^{2}}+k \frac{d x}{d t} & =x\left(F-2 x^{2}\right), \\
\frac{d F}{d t} & =\varepsilon .
\end{aligned}
$$

The initial value $F(0)$ is chosen smaller than a certain critical value $F_{c}$ for which the system exhibits a bifurcation. An example of a mechanical system that corresponds qualitatively with system (1.2) is the analogue of an elastic column, sketched in Figure 1 (see also Stoker (1950)).

If a slender straight elastic rod is subjected at its ends to compressive forces along the axis of the rod, the unbended equilibrium position is stable if the compressive forces are kept under a certain critical value. Beyond this value the column bends or buckles. We simplify the problem by considering an elastic system with only one degree of freedom. Two rods are connected by a hinge at point $C$ and are both free to slide along a vertical line at their other ends $A$ and $B$. The hinge $C$ is assumed to carry a particle of mass $m$. At the ends $A$ and $B$ forces $P$ act along the vertical line through $A$ and $B$. At point $C$ springs are placed, producing a sideways restoring force $f(x)$ depending upon the displacement $x$. Moreover, a restoring moment $M$ acts

* Received by the editors October 20, 1993; accepted for publication (in revised form) March 10, 1995. This research was supported by Nederlandse Organisatie voor Wetenschappelijk Onderzoek (NWO) grant 611-306-526.

† Department of Mathematics, Agricultural University, Dreijenlaan 4, 6703 HA Wageningen, the Netherlands. 

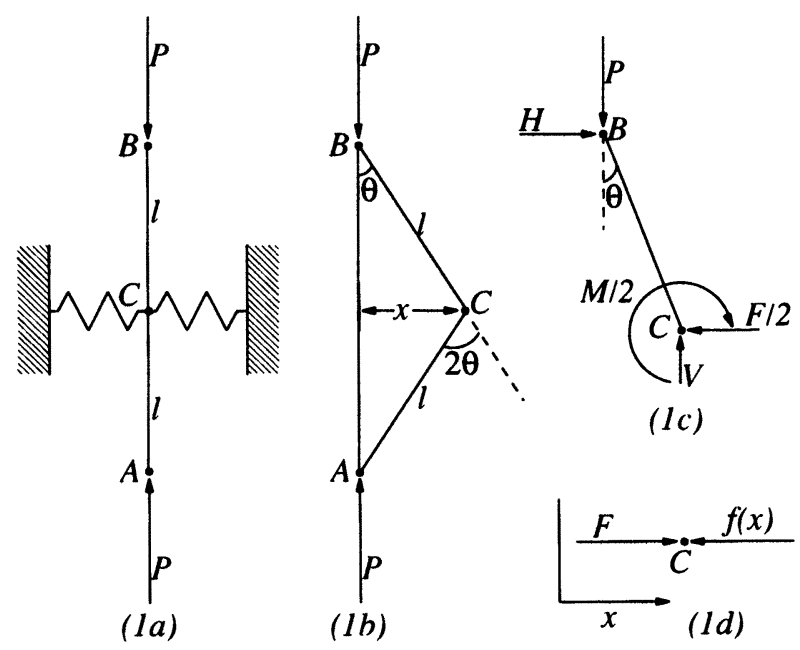

FIG. 1. Analogue of an elastic column.

upon the hinge. This moment is proportional to the angle $2 \theta$ between the two rods (see Figure 1(b)). In this way the bending stiffness of the continuous elastic column is simulated. Neglecting the damping and the mass of the rod, we obtain the following equations:

equilibrium of forces in the vertical direction:

$$
P=V .
$$

equation of moments about point $B$ :

$$
-\frac{M}{2}+V l \sin \theta-\frac{F l}{2} \cos \theta=0 .
$$

equation of motion of the mass $m$ :

$$
m \frac{d^{2} x}{d t^{2}}=F-f(x)
$$

For the lateral spring force we take $f(x)=\alpha x+\beta x^{3}$, with $\alpha$ and $\beta$ both positive constants, while for the restoring moment $M$ we have $M=2 K_{1} \theta$, with $K_{1}$ a constant. Assuming that the sideways displacement $x$ is sufficiently small so that terms with powers of $x / l$ higher than three can be ignored, we arrive at the following equation:

$$
m \frac{d^{2} x}{d t^{2}}+\left(\alpha+\frac{2 K_{1}}{l^{2}}-\frac{2 P}{l}\right) x+\left(\beta+\frac{4 K_{1}}{3 l^{4}}-\frac{P}{l^{3}}\right) x^{3}=0 .
$$

One solution is given by $x=0$. The question is whether this solution is stable when $P$ is increased. If $P$ is smaller than the critical value $P_{\text {crit }}$, a disturbance of the equilibrium position results in a small oscillation. We aim to predict the side to which the column will bend after $P$ has become larger than $P_{\text {crit }}$ while the oscillation is not yet damped.

For $F$ fixed smaller than $F_{c}$, the system (1.2) has one stable equilibrium. On a large time-scale the system shows a damped oscillation until $F$ has reached $F_{c}$. 
The parameter $F$ slowly passes $F_{c}$ corresponding to a transition from a simple stable equilibrium to one of the stable equilibria positioned on a parabolic curve: for $F$ fixed larger than $F_{c}$, the system has three equilibria, two of which are stable and one of which is unstable. Because $F$ is slowly increasing, the two stable equilibria are slowly varying in time. The solution in the neighbourhood of the stable slowly varying equilibria is approximated by a harmonic oscillation. For $F$ outside a certain $\varepsilon$-neighbourhood of $F_{c}$ asymptotic approximations are obtained with the aid of averaging methods. In order to describe the bifurcation, a local approximation, being a transition layer, has to be made. Local scaling analysis yields as an approximating differential equation the second Painleve transcendent. In order to connect local solutions that are valid before, during, and after the transition, asymptotic matching techniques have to be used and an extension theorem formulated by Eckhaus (1979) has to be applied.

It is remarked that existence and uniqueness of solutions of (1.2) is guaranteed for $t \in[-M / \varepsilon, M / \varepsilon]$, with $M$ an arbitrary large positive number independent of $\varepsilon$. Such a proof of existence and uniqueness can be based on the energy integral of the system; see also Chillingworth (1976, pp. 187-188). In Lemma 5.1 we will use the same method to prove the validity of the approximation near the bifurcation point.

Haberman (1979) also studied this type of nonlinear differential equations. He studied systems without damping and used the method of eliminating secular terms. In a similar way we apply averaging to eliminate secular terms and, in contrast to Haberman, we study systems with damping. There are obvious discrepancies in the computed values of coefficients of some asymptotic series. More relevant is that we prove the validity of the matched asymptotic approximations and predict the state of the system after passing the bifurcation point from the initial state. This result almost looks like prediction of the outcome of flipping a coin. Neishtadt $(1987,1988)$ and Baer, Erneux, and Rinzel (1989) concentrate on the slow passage through a Hopf bifurcation from a stable steady state to a stable time-periodic solution and demonstrate that this case is quite different from a steady bifurcation or limit point. The transition from an oscillatory solution to steady state has been investigated by Holden and Erneux (1993). The case of second-order jump phenomena with damping has been studied by Marée (1993).

In this study the potential takes the form of a double-well potential after bifurcation. We assume that the trajectory is close to the equilibrium before bifurcation. Therefore, it has directly been captured in either well after bifurcation. This means that the unperturbed separatrix that appears after passing the bifurcation point will not be crossed. The problem of the crossing of a separatrix by nonlinear oscillations that correspond to a slowly varying potential which remains double-welled has been analysed by Bourland and Haberman (1990, 1994), Neishtadt (1986, 1993), and Henrard (1993). In the asymptotic limit $(\varepsilon \rightarrow 0)$ the capture problem becomes probabilistic and predictions become pointless in view of the extreme sensitivity to initial data. The work of Bourland, Haberman, and Kath (1991) contains formal averaging formulas similar to those of this study. These are only valid away from the bifurcation point. Denier and Grimshaw (1988) also study nonlinear differential equations with a slowly varying parameter. They find that, depending on the initial amplitudes, the solutions of the transition equations either are asymptotically equivalent to the bifurcated solutions or develop algebraic singularities at some positive time.

In $\S 2$ we consider the different equilibrium states of the system when the parameter $F$ is fixed. In $\S 3$ the solution is approximated by averaging for an interval in which the solution is sufficiently bounded away below the bifurcation point, whereas in $\S 4$ we obtain an asymptotic approximation for the solution beyond the critical 
point. Moreover, it is shown that these approximations remain valid outside an $\varepsilon^{2 / 3}$ neighbourhood of the critical point $F=F_{c}$. In $\S 5$ we analyse the transition layer equation and obtain matching conditions for this local asymptotic solution. Furthermore, it is proven that the transition layer solution is a local approximation of the exact solution. This local solution has an overlap with the other (outer) approximations. In $\S 6$ we formulate an important result which connects the integration constants in the averaged asymptotic solution below criticality with those in the one above criticality. In this way we obtain a remarkable result: one can find asymptotic approximations for predicting the behaviour of solutions after passing the bifurcation point at the basis of the initial values. In $\S 7$ we consider the general class of bifurcation problems, of which the present problem is a special case. Finally, in $\S 8$, we make some concluding remarks.

2. The reduced system with fixed parameter. Substitution of $\varepsilon=0$ in (1.2) yields the reduced system

$$
\begin{gathered}
\frac{d^{2} x}{d t^{2}}-x\left(F-2 x^{2}\right)=0, \\
\frac{d F}{d t}=0 .
\end{gathered}
$$

For $F$ fixed and smaller than the critical value

$$
F_{c}=0,
$$

the reduced system (2.2) has one equilibrium (for which $d x / d t=0$ ):

$$
x_{0}=0 .
$$

Linearization at $\left(x_{0}, 0\right)$ yields a system with an equilibrium being a centre point. Using the lemma of Morse (see Verhulst (1990)) it is clear that for the nonlinear system (2.1a) this point is also a centre point. When $F=F_{c}$, the unique equilibrium $x=x_{c}$ is still stable. For $F>F_{c}$ it becomes an unstable point of saddle-point type. Moreover the system then exhibits two "supercritical" stable equilibria (for which $y=0)$ :

$$
x_{-1}=-\sqrt{\frac{F}{2}}, \quad x_{1}=\sqrt{\frac{F}{2}} .
$$

Haberman (1979) calls the transition from a stable line to a parabolic arc, as $F$ passes $F_{c}$, a "parabolic bifurcation." In literature this phenomenon has commonly been called a pitchfork bifurcation. This pitchfork bifurcation is illustrated in Figures 2 and 3 . For $\varepsilon=0$ the energy integral of the system (1.2) equals

$$
E=\frac{1}{2} y^{2}-\frac{1}{2} F x^{2}+\frac{1}{2} x^{4} .
$$

3. Asymptotic expansion valid before passage of the bifurcation point. We construct an asymptotic solution to the initial value problem (1.2) valid outside a certain $\varepsilon$-neighbourhood of $F=0$, corresponding to initial conditions close to the outer equilibrium solution $x=x_{0}=0$. We consider perturbations of the equilibrium of the form

$$
x(t)=\sqrt{\varepsilon} u(t) .
$$




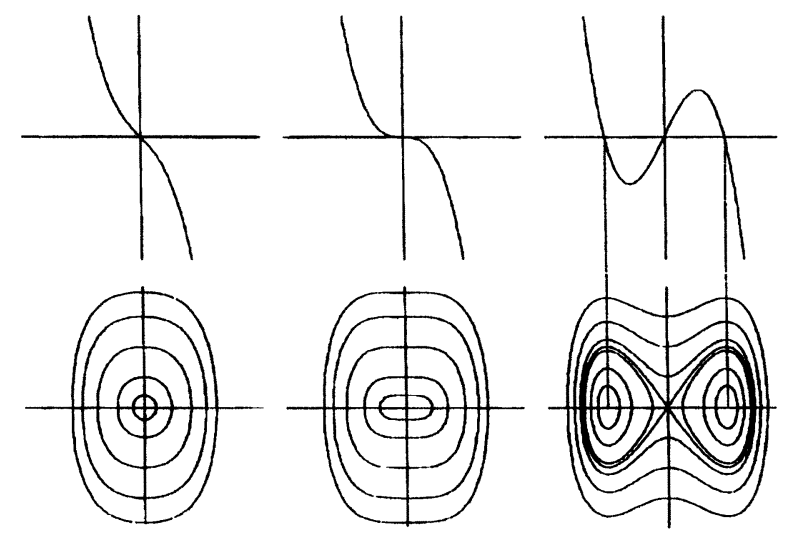

FIG. 2. The graph of the function $f(x)=x\left(F-2 x^{2}\right)$ in three cases of fixed $F$ and the corresponding phase portrait of (2.1a) for different values of $F$. From left to right, $F<F_{c}, F=F_{c}$, and $F>F_{c}$.

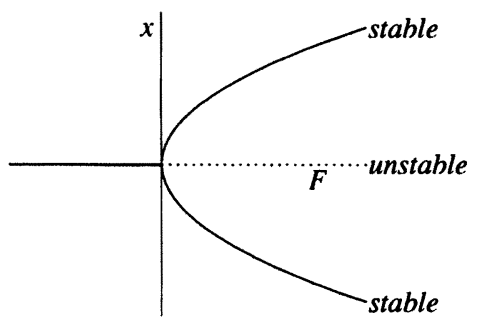

FIG. 3. The branches of the limit solution as a function of $F$.

Furthermore, we take as initial values

$$
\begin{aligned}
x(0) & =\sqrt{\varepsilon} a, \\
\frac{d x}{d t}(0) & =\sqrt{\varepsilon} b, \\
F(0) & =F_{0}<F_{c}=0 .
\end{aligned}
$$

Substitution in $(1.2 \mathrm{a})$ yields

$$
\frac{d^{2} u}{d t^{2}}+\omega^{2}(F) u=-\kappa \varepsilon \frac{d u}{d t}-2 \varepsilon u^{3}
$$

with

$$
\omega^{2}(F)=-F
$$

The solution remains for a certain time in a $\sqrt{ } \varepsilon$-neighbourhood of $x_{0}=0$. Using the transformations

$$
\begin{gathered}
u=e^{-\frac{1}{2} \kappa \varepsilon t} w:=r_{1} w \\
w=\omega^{-\frac{1}{2}}(F) y
\end{gathered}
$$




$$
\begin{gathered}
\frac{d y}{d t}=\omega(F) v \\
\frac{d v}{d t}=\frac{1}{\omega(F)} \frac{d^{2} y}{d t^{2}}-\frac{\varepsilon}{\omega(F)} \frac{d \omega}{d F} v
\end{gathered}
$$

introducing polar coordinates

$$
y=r \sin \varphi, \quad v=r \cos \varphi
$$

with initial values

$$
y(0)=r_{0} \sin \varphi_{0}, \quad u(0)=r_{0} \cos \varphi_{0}
$$

transforming the time-scale

$$
\tau=\int_{F_{0}}^{F} \frac{\omega(F) d F}{\varepsilon}
$$

or

$$
F=-\left(\left(-F_{0}\right)^{\frac{3}{2}}-\frac{3 \varepsilon}{2} \tau\right)^{\frac{2}{3}}
$$

eliminating $F$; and setting

$$
\psi=\varphi-\tau,
$$

we finally arrive at the following initial value problem:

$$
\begin{aligned}
\frac{d r_{1}}{d \tau}= & \frac{-\kappa \varepsilon}{2 \omega(\tau, \varepsilon)} r_{1}, \quad \omega(\tau, \varepsilon)=\left(\left(-F_{0}\right)^{\frac{3}{2}}-\frac{3 \varepsilon}{2} \tau\right)^{\frac{1}{3}}, \quad r_{1}(0)=1 \\
\frac{d r}{d \tau}= & \frac{\varepsilon}{\omega^{3}(\tau, \varepsilon)}\left(-2 r_{1}^{2} r^{3} \sin ^{3}(\psi+\tau) \cos (\psi+\tau)\right. \\
& \left.+\frac{1}{4} \kappa^{2} r \varepsilon \omega(\tau \varepsilon) \sin (\psi+\tau) \cos (\psi+\tau)\right) \\
& +\frac{1}{\omega^{2}(\tau, \varepsilon)}\left(\frac{d \omega}{d \tau}\right)^{2}\left(-\frac{3}{4} r \sin (\psi+\tau) \cos (\psi+\tau)\right) \\
& +\frac{1}{2} \omega^{-1}(\tau, \varepsilon) \frac{d^{2} \omega}{d \tau^{2}} r \sin (\psi+\tau) \cos (\psi+\tau), \\
\frac{d \psi}{d t}= & \frac{\varepsilon}{\omega^{3}(\tau, \varepsilon)}\left(2 r_{1}^{2} r^{2} \sin ^{4}(\psi+\tau)-\frac{1}{4} \kappa^{2} \varepsilon \omega(\tau, \varepsilon) \sin ^{2}(\psi+\tau)\right) \\
& +\frac{1}{\omega^{2}(\tau, \varepsilon)}\left(\frac{d \omega}{d \tau}\right)^{2}\left(\frac{3}{4} \sin ^{2}(\psi+\tau)\right) \\
& -\frac{1}{2} \omega^{-1}(\tau, \varepsilon) \frac{d^{2} \omega}{d \tau^{2}} \sin ^{2}(\psi+\tau) .
\end{aligned}
$$

From (3.8b) we conclude that $\omega(\tau, \varepsilon)=0$ for $\tau=\tau_{0}$ with

$$
\tau_{0}=\frac{2}{3 \varepsilon}\left(-F_{0}\right)^{\frac{3}{2}} .
$$


With the aid of the lemma of Gronwall we will prove an approximation theorem for $\tau \in\left[0, \tau_{0}-\delta^{-1}(\varepsilon)\right]$ and thus for $F \in\left[F_{0},-\left(3 \varepsilon \delta^{-1}(\varepsilon) / 2\right)^{2 / 3}\right]$ with $\delta(\varepsilon)=o(1)$ an asymptotic order function.

LEMMA 3.1 (Gronwall). Suppose, for $t_{0} \leq t \leq t_{0}+a$, that

$$
\varphi(t) \leq \delta_{2}\left(t-t_{0}\right)+\delta_{1} \int_{t_{o}}^{t} \varphi(s) d s+\delta_{3}
$$

with $\varphi(t)$ continuous and $\varphi(t) \geq 0$ for $t_{0} \leq t \leq t_{0}+a$, where $\delta_{1}, \delta_{2}$, and $\delta_{3}$ are constants with $\delta_{1}>0, \delta_{2} \geq 0$, and $\delta_{3} \geq 0$. Then for $t_{0} \leq t \leq t_{0}+a$

$$
\varphi(t) \leq\left(\frac{\delta_{2}}{\delta_{1}}+\delta_{3}\right) e^{\delta_{1}\left(t-t_{0}\right)}-\frac{\delta_{2}}{\delta_{1}} .
$$

A proof of this lemma is given by Sanders and Verhulst (1985).

We now state an approximation theorem

THEOREM 3.2. Consider the initial value problem

$$
\begin{aligned}
& \frac{d r_{1}}{d \tau}=\frac{-\kappa \varepsilon}{2 \omega(\tau, \varepsilon)} r_{1} ; \quad \omega(\tau, \varepsilon)=\left(\left(-F_{0}\right)^{\frac{3}{2}}-\frac{3 \varepsilon}{2} \tau\right)^{\frac{1}{3}}, \quad r_{1}(0)=1 \\
& \frac{d r}{d \tau}=\frac{\varepsilon}{\omega^{3}(\tau, \varepsilon)}\left(g_{1}\left(r_{1}, r, \psi, \tau\right)\right. \\
& \left.+\varepsilon \omega(\tau, \varepsilon) h_{1}(r, \psi, \tau)\right)+\frac{1}{\omega^{2}(\tau, \varepsilon)}+\left(\frac{d \omega}{d \tau}\right)^{2} m_{1}(r, \psi, \tau) \\
& +\frac{1}{\omega(\tau, \varepsilon)} \frac{d^{2} \omega}{d \tau^{2}} l_{1}(r, \psi, \tau), \quad r(0)=r_{0}, \\
& \frac{d \psi}{d \tau}=\frac{\varepsilon}{\omega^{3}(\tau, \varepsilon)}\left(g_{2}\left(r_{1}, r, \psi, \tau\right)+\varepsilon \omega(\tau, \varepsilon) h_{2}(r, \psi, \tau)\right) \\
& +\frac{1}{\omega^{2}(\tau, \varepsilon)}\left(\frac{d \omega}{d \tau}\right)^{2} m_{2}(r, \psi, \tau) \\
& +\frac{1}{\omega(\tau, \varepsilon)} \frac{d^{2} \omega}{d \tau^{2}} l_{2}(r, \psi, \tau), \quad \psi(0)=\varphi_{0},
\end{aligned}
$$

for $0 \leq \tau<\tau_{0}$.

Suppose that

a) the vector functions $g, h, m, l, \nabla g$, and $\nabla h$ are continuous in $\tau$ and in $x=$ $\left(r_{1}, r, \psi\right)^{t}$ or $y=(r, \psi)^{t}$ and bounded by a constant $M$ independent of $\varepsilon$;

b) $m$ and $l$ are Lipschitz continuous in $y=(r, \psi)^{t}$ with Lipschitz constant L;

c) $g$ and $h$ are $T$-periodic in $\tau$ with averages $g^{\circ}$ and $h^{\circ}$.

Let $\left(r_{1}, r, \psi\right)$ be the solution of this system. Moreover, let $\left(r_{1 a}, r_{a}, \psi_{a}\right)$ be the solution of the $\tau$-averaged system

$$
\begin{aligned}
& \frac{d r_{1 a}}{d \tau}=\frac{-\kappa \varepsilon}{2 \omega(\tau, \varepsilon)} r_{1 a}, \quad r_{1 a}(0)=1, \\
& \frac{d r_{a}}{d \tau}=\frac{\varepsilon}{\omega^{3}(\tau, \varepsilon)}\left(g_{1}^{0}\left(r_{1 a}, r_{a}, \psi_{a}\right)+\varepsilon \omega(\tau, \varepsilon) h_{1}^{0}\left(r_{a}, \psi_{a}\right)\right), \quad r_{a}(0)=r_{0} \\
& \frac{d \psi_{a}}{d \tau}=\frac{\varepsilon}{\omega^{3}(\tau, \varepsilon)}\left(g_{2}^{0}\left(r_{1 a}, r_{a}, \psi_{a}\right)+\varepsilon \omega(\tau, \varepsilon) h_{2}^{0}\left(r_{a}, \psi_{a}\right)\right), \quad \psi_{a}(0)=\varphi_{0}
\end{aligned}
$$


Let $\delta(\varepsilon)$ be an asymptotic order function satisfying $\delta(\varepsilon)=o(1)$ as well as $\varepsilon=$ $O(\delta(\varepsilon))$. Then for $\tau \in\left[0, \tau_{0}-\delta^{-1}(\varepsilon)\right]$

$$
r_{1}(\tau)=r_{1 a}(\tau), \quad r(\tau)=r_{a}(\tau)+o(1), \quad \psi(\tau)=\psi_{a}(\tau)+o(1) .
$$

Remark 1. For a vector function $f \in \mathbb{R}^{n}$ with components $f_{i}, i=1, \ldots, n$, we will use the norm

$$
\|f\|=\sum_{i=1}^{n}\left|f_{i}\right| .
$$

Remark 2. The time-scale of $\tau_{0}$, which has been determined as a zero of $\omega$, is of order $O(1 / \varepsilon) . \delta(\varepsilon)$ is such that $\tau_{0}-\delta^{-1}(\varepsilon) \geq 0$.

Proof. We immediately obtain

$$
r_{1 a}(\tau)=r_{1}(\tau)=\exp \left\{\frac{\kappa}{2}\left(\left(-F_{0}\right)^{\frac{3}{2}}-\frac{3 \varepsilon}{2} \tau\right)^{\frac{2}{3}}+\frac{\kappa}{2} F_{0}\right\}
$$

so

$$
0 \leq r_{1 a}(\tau) \leq 1 \text { for } 0 \leq \tau<\tau_{0} .
$$

Furthermore, for $\tau \in\left[0, \tau_{0}-\delta^{-1}(\varepsilon)\right]$,

$$
\begin{aligned}
& \left(-F_{0}\right)^{-\frac{1}{2}} \leq \omega^{-1}(\tau, \varepsilon) \leq\left(\frac{3}{2} \varepsilon \delta^{-1}(\varepsilon)\right)^{-\frac{1}{3}}, \\
& \frac{\varepsilon}{2}\left(F_{0}\right)^{-1} \leq\left|\frac{d \omega}{d \tau}\right| \leq \frac{\varepsilon}{2}\left(\frac{3}{2} \varepsilon \delta^{-1}(\varepsilon)\right)^{-\frac{2}{3}} .
\end{aligned}
$$

We define

$$
x=\left(r_{1}, r, \psi\right)^{t}=\left(x_{1}, x_{2}\right)^{t} \quad \text { with } x_{1}=r_{1} \text { and } x_{2}=(r, \psi)^{t}
$$

and introduce

$$
u^{1}(x, \tau)=\int_{0}^{\tau}\left[g(x, s)-g^{\circ}(x)+\varepsilon \omega(s, \varepsilon)\left(h\left(x_{2}, s\right)-h^{0}\left(x_{2}\right)\right)\right] d s .
$$

This integral is bounded: $\left\|u^{1}(x, \tau)\right\| \leq 4 M T$. Now a "near-identity" transformation is applied:

$$
x_{2}(\tau)=z_{2}(\tau)+\frac{\varepsilon}{\omega^{3}(\tau, \varepsilon)} u^{1}(z(\tau), \tau) .
$$

This is a "near identity" because

$$
\frac{\varepsilon}{\omega^{3}(\tau, \varepsilon)}=O(\delta(\varepsilon)) \quad \text { for } \tau \in\left[0, \tau_{0}-\delta^{-1}(\varepsilon)\right] .
$$

Differentiating (3.22) we obtain with (3.15) and with the aid of (3.21)

$$
\left[E+\frac{\varepsilon}{\omega^{3}(\tau, \varepsilon)} \frac{\partial}{\partial z_{2}} u^{1}(z(\tau), \tau)\right] \frac{d z_{2}}{d \tau}=\frac{\varepsilon}{\omega^{3}(\tau, \varepsilon)}\left(g^{\circ}(z)+\varepsilon \omega(\tau, \varepsilon) h^{\circ}(z)\right)+R
$$


with $R$ a vector function of $z, \tau$, and $\varepsilon$. This remainder term $R$ is estimated as follows:

$$
R=O\left(\delta^{2}(\varepsilon)\right) .
$$

The matrix $E$ in (3.23) is the unity matrix. Since both $\partial u^{1} / \partial z_{2}$ and $u^{1}$ are bounded. we can invert

$$
\begin{aligned}
{[E} & \left.+\frac{\varepsilon}{\omega^{3}(\tau, \varepsilon)} \frac{\partial}{\partial z_{2}} u^{1}(z(\tau), \tau)\right]^{-1} \\
& =E-\frac{\varepsilon}{\omega^{3}(\tau, \varepsilon)} \frac{\partial}{\partial z_{2}} u^{1}(z(\tau), \tau)+O\left(\frac{\varepsilon^{2}}{\omega^{6}(\tau, \varepsilon)}\right) .
\end{aligned}
$$

So from (3.23), we obtain the following equation for $z_{2}$ :

$$
\frac{d z_{2}}{d \tau}=\delta_{1}(\varepsilon)\left(g^{\circ}(z)+\varepsilon \omega(\tau, \varepsilon) h^{\circ}\left(z_{2}\right)\right)+\delta_{2}(\varepsilon)
$$

with

$$
\delta_{1}(\varepsilon)=\varepsilon \omega^{-3}(\tau, \varepsilon)=O(\delta(\varepsilon)) \quad \text { and } \quad \delta_{2}(\varepsilon)=O\left(\delta^{2}(\varepsilon)\right)=o\left(\delta_{1}(\varepsilon)\right) .
$$

The solution of the averaged system

$$
\frac{d x_{2 a}}{d \tau}=\frac{\varepsilon}{\omega^{3}(\tau, \varepsilon)}\left(g^{\circ}\left(x_{a}\right)+\varepsilon \omega(\tau, \varepsilon) h^{\circ}\left(x_{2 a}\right)\right), \quad x_{2 a}(0)=z_{2}(0),
$$

approximates the solution of (3.26) for $\tau \in\left[0, \tau_{0}-\delta^{-1}(\varepsilon)\right]$ in the following way:

$$
z_{2}(\tau)=x_{2 a}(\tau)+o(1)
$$

for

$$
\begin{aligned}
\left\|z_{2}(\tau)-x_{2 a}(\tau)\right\|= & \left\|\int_{0}^{\tau} \frac{d z_{2}}{d \tau} d \tau-\int_{0}^{\tau} \frac{d x_{2 a}}{d \tau} d \tau\right\| \\
\leq & \| \delta_{1}(\varepsilon) \int_{0}^{\tau}\left(g^{\circ}(z)-g^{\circ}\left(x_{a}\right)\right. \\
& \left.+\varepsilon \omega(\tau, \varepsilon)\left(h^{\circ}\left(z_{2}\right)-h^{\circ}\left(x_{2 a}\right)\right)\right) \delta \tau \|+\delta_{2} \varepsilon \tau \\
\leq & 2 L \delta_{1}(\varepsilon) \tau\left\|z-x_{a}\right\|+\delta_{2} \varepsilon \tau \quad\left(z_{1}=x_{1 a}\right) .
\end{aligned}
$$

Application of the lemma of Gronwall now completes the proof:

$$
\begin{aligned}
\left\|z_{2}(\tau)-x_{2 a}(\tau)\right\| & \leq \frac{\delta_{2}}{2 L \delta_{1}} e^{2 L \delta_{1} \tau}=O(\delta(\varepsilon)) \\
& =o(1) \text { for } \tau \in\left[0, \tau_{0}-\delta^{-1}(\varepsilon)\right] .
\end{aligned}
$$

This yields an asymptotic expansion of the solution of (1.2) in the neighbourhood of $x_{0}$ for $F<0$ and $\varepsilon^{2 / 3}=o(F)$.

Corollary 3.3. For $F_{0}<F<0$ and $\varepsilon^{2 / 3}=o(F)$ the solution of (1.2) has the following expansion:

$$
x(t)=\sqrt{\varepsilon} r_{a} r_{1 a} \omega^{-\frac{1}{2}}(F) \sin \left(\tau+\psi_{a}\right)+o\left(\sqrt{\varepsilon} \omega^{-\frac{1}{2}}(F)\right),
$$


with $\left(r_{1 a}, r_{1}, \psi_{a}\right)$ the solution of the system

$$
\begin{gathered}
\frac{d r_{1 a}}{d \tau}=\frac{-\kappa \varepsilon}{2 \omega(\tau, \varepsilon)} r_{1 a}, \quad r_{1 a}(0)=1, \\
\frac{d r_{a}}{d \tau}=0, \quad r_{a}(0)=r_{0}, \\
\frac{d \psi_{a}}{d \tau}=\frac{\varepsilon}{\omega^{3}(\tau, \varepsilon)}\left(\frac{3}{4} r_{1 a}^{2} r_{a}^{2}-\frac{1}{8} \kappa^{2} \omega(\tau, \varepsilon) \varepsilon\right), \quad \psi_{a}(0)=\varphi_{0},
\end{gathered}
$$

with $\omega(\tau, \varepsilon)$ as defined in $(3.14 \mathrm{a})$.

Proof. Problems arise when the angular velocity $\omega(F)$ tends to zero which is tied up with boundary layer behaviour in the neighbourhood of $F=0$. An approximation for the bifurcation time is therefore $t_{0}=-F_{0} \varepsilon^{-1}$. Because (3.10) is a special case of the initial value problem stated in Theorem 3.2, the averaged system (3.32) is an $o(1)$ approximation of the complete system (3.10) for $\tau \in\left[0 . \tau_{0}-\delta^{-1}(\varepsilon)\right]$ with $\delta(\varepsilon)=o(1)$ and $\delta(\varepsilon)=O(\varepsilon)$. The result is summarized as follows: (3.32), being an approximation of the solution of (1.2), is valid when $F_{0}<F<0$ and $\varepsilon^{\frac{2}{3}}=o(F)$.

4. Asymptotic expansion valid after passage of the bifurcation point. We analyse the solution to the initial value problem (1.2) for $F \geq F_{c}+\delta\left(F_{c}=0\right)$ close to one of the two stable outer equilibrium solutions $x=x_{ \pm 1}$. Because of the symmetry of the problem it is sufficient to consider the perturbation of one of the equilibria of the reduced system: we choose $x=x_{1}=\sqrt{ }(F / 2)$. To analyse a "slowly varying equilibrium solution" $x_{1 s v}(F)$ we rewrite (1.2):

$$
\frac{d^{2} x}{d t^{2}}=\varepsilon^{2} \frac{d^{2} x}{d F^{2}}=-\kappa \varepsilon^{2} \frac{d x}{d F}+g(x, F)
$$

with

$$
g(x, F)=x\left(F-2 x^{2}\right) .
$$

Assuming that the derivatives in (4.1) are small, we obtain a "slowly varying equilibrium solution" for $F>F_{c}=0$ by perturbing the dependent variable $x$ at $x_{1}(F)=$ $\sqrt{ }(F / 2)$. Because $g\left(x_{1}, F\right)=0$, we can use the Taylor series of $g(x, F)$ at $x=x_{1}(F)$. This changes (4.1) to

$$
\varepsilon^{2} \frac{d^{2} x}{d F^{2}}=-\kappa \varepsilon^{2} \frac{d x}{d F}+\left.\left(x-x_{1}\right) \frac{\partial g}{\partial x}\right|_{x_{1}}+\left.\frac{\left(x-x_{1}\right)^{2}}{2} \frac{\partial^{2} g}{\partial x^{2}}\right|_{x_{1}}+\left.\frac{\left(x-x_{1}\right)^{3}}{6} \frac{\partial^{3} g}{\partial x^{3}}\right|_{x_{1}}
$$

In this way we obtain an asymptotic expansion for the "slowly varying equilibrium solution" of (4.1):

$$
x_{1 s v}(F)=\sqrt{\frac{F}{2}}-\frac{1}{16} \kappa \varepsilon^{2}\left(\frac{F}{2}\right)^{-\frac{3}{2}}+\frac{1}{64} \varepsilon^{2}\left(\frac{F}{2}\right)^{-\frac{5}{2}}+\cdots .
$$

From Theorem 9.1 formulated by Verhulst (1990) it follows that this is the asymptotic expansion of the solution. For a solution that holds in a $\sqrt{ } \varepsilon$-neighbourhood of this slowly varying solution we write

$$
x(t)=x_{1 s v}(F)+\sqrt{\varepsilon} u(t) .
$$


Furthermore, we assume that the solution takes the following values for $t=t_{1}=$ $-F_{0} \varepsilon^{-1}+M \varepsilon^{-1}$ with $M$ an $\varepsilon$-independent positive constant.

$$
\begin{gathered}
x\left(t_{1}\right)=x_{1}\left(F_{1}\right)+\sqrt{\varepsilon} a_{1}, \\
\frac{d x}{d t}\left(t_{1}\right)=\frac{d x_{1}}{d t}\left(F_{1}\right)+\sqrt{\varepsilon} b_{1}, \\
F\left(t_{1}\right)=F_{1}=\varepsilon t_{1},
\end{gathered}
$$

so the solution is at $t=t_{1}$ "far away" from the bifurcation time $t=-F_{0} \varepsilon^{-1}$. Substitution in (1.2) yields

$$
\begin{gathered}
\frac{d^{2} u}{d t^{2}}+\omega_{1}^{2}(F) u=-6 \sqrt{\varepsilon} u^{2} x_{1 s v}(F)-\kappa \varepsilon \frac{d u}{d t}-2 \varepsilon u^{3}, \\
\frac{d F}{d t}=\varepsilon
\end{gathered}
$$

with

$$
\omega_{1}^{2}(F)=6 x_{1 s v}^{2}-F
$$

In the same way as in $\S 3$ we now carry out the transformations

$$
\begin{gathered}
u=e^{-\frac{1}{2} \kappa \varepsilon t} w:=r_{1}^{*} w \\
w=\omega_{1}^{-\frac{1}{2}}(F) y, \\
\frac{d y}{d t}=\omega_{1}(F) v, \\
\frac{d v}{d t}=\frac{1}{\omega_{1}^{2}(F)} \frac{d^{2} y}{d t^{2}}-\frac{\varepsilon}{\omega_{1}(F)} \frac{d \omega_{1}}{d F} v
\end{gathered}
$$

and introduce polar coordinates

$$
y=r^{*} \cos \varphi^{*}, \quad v=-r^{*} \sin \varphi^{*}
$$

with values at $t_{1}$

$$
y\left(t_{1}\right)=r_{0}^{*} \cos \varphi_{0}^{*}, \quad u\left(t_{1}\right)=-r_{0}^{*} \sin \varphi_{0}^{*} .
$$

Next, we make the transformation

$$
\tau=\int_{F}^{F_{1}} \frac{\omega_{1}(F) d F}{\varepsilon}
$$

and set

$$
\psi^{*}=\varphi^{*}+\tau
$$


so we finally arrive at the following system:

$$
\frac{d r_{1}^{*}}{d \tau}=\frac{\kappa \varepsilon}{2 \omega_{1}(F)} r_{1}^{*}, \quad r_{1}^{*}\left(t_{1}\right)=e^{-\frac{1}{2} \kappa \varepsilon t_{1}}
$$

$$
\frac{d F}{d \tau}=\frac{-\varepsilon}{\omega_{1}(F)}, \quad F\left(t_{1}\right)=F_{1}
$$

$$
\begin{aligned}
\frac{d r^{*}}{d \tau}= & -\sqrt{\varepsilon}\left(6 \omega_{1}^{-\frac{5}{2}}(F) r_{1}^{*} r^{*^{2}} \cos ^{2}\left(\psi^{*}+\tau\right) \sin \left(\psi^{*}+\tau\right) x_{1 s v}(F)\right) \\
& -2 \varepsilon \omega_{1}^{-3}(F) r_{1}^{*^{2}} r^{*^{3}} \cos ^{3}\left(\psi^{*}+\tau\right) \sin \left(\psi^{*}+\tau\right) \\
& -\varepsilon^{2}\left(-\frac{1}{4} \kappa^{2} \omega_{1}^{-2}(F) r^{*} \cos \left(\psi^{*}+\tau\right) \sin \left(\psi^{*}+\tau\right)\right. \\
& +\frac{3}{4} \omega_{1}^{-4}(F) r^{*} \cos \left(\psi^{*}+\tau\right) \sin \left(\psi^{*}+\tau\right)\left(\frac{d \omega_{1}}{d F}\right)^{2} \\
& \left.+\frac{1}{2} \omega_{1}^{-3}(F) r^{*} \cos \left(\psi^{*}+\tau\right) \sin \left(\psi^{*}+\tau\right) \frac{d^{2} \omega_{1}}{d F^{2}}\right), \quad r^{*}\left(t_{1}\right)=r_{0}^{*}
\end{aligned}
$$

$$
\begin{aligned}
\frac{d \psi^{*}}{d \tau}= & -\sqrt{\varepsilon}\left(6 \omega_{1}^{-\frac{5}{2}}(F) r_{1}^{*} r^{*} \cos ^{3}\left(\psi^{*}+\tau\right) x_{1 s v}(F)\right) \\
& -2 \varepsilon \omega_{1}^{-3}(F) r_{1}^{*^{2}} r^{*^{2}} \cos ^{4}\left(\psi^{*}+\tau\right) \\
& -\varepsilon^{2}\left(-\frac{1}{4} \kappa^{2} \omega_{1}^{-2}(F) \cos ^{2}\left(\psi^{*}+\tau\right)+\frac{3}{4} \omega_{1}^{-4}(F) \cos ^{2}\left(\psi^{*}+\tau\right)\left(\frac{d \omega_{1}}{d F}\right)^{2}\right. \\
& \left.+\frac{1}{2} \omega_{1}^{-3}(F) \cos ^{2}\left(\psi^{*}+\tau\right) \frac{d^{2} \omega_{1}}{d F^{2}}\right), \quad \psi^{*}\left(t_{1}\right)=\varphi_{0}^{*} .
\end{aligned}
$$

We will consider

$$
0<\delta_{1}(\varepsilon) \leq F<F_{1}=O(1)
$$

with

$$
\varepsilon^{\frac{2}{3}}=o\left(\delta_{1}(\varepsilon)\right)
$$

We now obtain the following approximations:

$$
\begin{gathered}
x_{l s v}(F)=\sqrt{\frac{F}{2}}+O\left(\varepsilon^{2} F^{-\frac{5}{2}}\right), \\
\omega_{1}(F)=\sqrt{2 F}\left(1+O\left(\varepsilon^{2} F^{-3}\right)\right), \\
\omega_{1}^{-a}(F)=(2 F)^{-\frac{a}{2}}\left(1+O\left(\varepsilon^{2} F^{-3}\right)\right), \quad a \in \mathbb{R}, \\
\tau=\frac{1}{\varepsilon}\left(\frac{1}{3}(2 F)^{\frac{3}{2}}+O\left(\varepsilon^{2} F^{-\frac{3}{2}}\right)\right),
\end{gathered}
$$


so

$$
\frac{1}{\delta_{1}^{*}(\varepsilon)} \leq \tau \leq \frac{1}{\delta_{1}^{*}(\varepsilon)} \text { with } \delta_{1}^{*}(\varepsilon)=o(1) \text { and } \delta_{2}^{*}(\varepsilon)=O(\varepsilon) .
$$

System (4.13) then transforms into

$$
\begin{gathered}
\frac{d r_{1}^{*}}{d \tau}=\frac{\kappa \varepsilon}{2 \sqrt{2 F}} r_{1}^{*}+O\left(\varepsilon^{3} F^{-\frac{7}{2}}\right) \\
\frac{d F}{d \tau}=\frac{-\varepsilon}{\sqrt{2 F}}+O\left(\varepsilon^{3} F^{-\frac{7}{2}}\right) \\
\frac{d r^{*}}{d \tau}=\frac{-\sqrt{\varepsilon} \cdot 2^{-\frac{7}{4}}}{F^{\frac{3}{4}}}\left(6 r_{1}^{*} r^{*^{2}} \cos ^{2}\left(\psi^{*}+\tau\right) \sin \left(\psi^{*}+\tau\right)\right) \\
-\frac{\varepsilon}{(2 F)^{\frac{3}{2}}}\left(2 r_{1}^{*^{2}} r^{*^{3}} \cos ^{3}\left(\psi^{*}+\tau\right) \sin \left(\psi^{*}+\tau\right)\right) \\
-\frac{\varepsilon^{2}}{2 F}\left(-\frac{1}{4} \kappa^{2} r^{*} \cos ^{*}\left(\psi^{*}+\tau\right) \sin \left(\psi^{*}+\tau\right)\right)+O\left(\varepsilon^{2} F^{-3}\right), \\
\frac{d \psi^{*}}{d \tau}=-\frac{\sqrt{\varepsilon} \cdot 2^{-\frac{7}{4}}}{F^{\frac{3}{4}}}\left(6 r_{1}^{*} r^{*} \cos ^{3}\left(\psi^{*}+\tau\right)\right)-\frac{\varepsilon}{(2 F)^{\frac{3}{2}}}\left(2 r_{1}^{*^{2}} r^{*^{2}} \cos ^{4}\left(\psi^{*}+\tau\right)\right) \\
-\frac{\varepsilon^{2}}{2 F}\left(-\frac{1}{4} \kappa^{2} \cos ^{2}\left(\psi^{*}+\tau\right)\right)+O\left(\varepsilon^{2} F^{-3}\right) .
\end{gathered}
$$

It follows from

$$
r_{1}^{*}=\exp \{-\kappa \varepsilon / 2 t\} \quad \text { that } 0 \leq r_{1}^{*} \leq 1 \text { for } t \in\left[-F_{0} \varepsilon^{-1}, t_{1}\right] .
$$

With the aid of the lemma of Gronwall we will now prove a "second-order" approximation theorem; we will obtain an estimate of $O\left(\varepsilon F^{-3 / 2}\right)$ taking into account both the $O\left(\varepsilon^{1 / 2} F^{-3 / 4}\right)$ terms and the $O\left(\varepsilon F^{-3 / 2}\right)$ terms of (4.16). Note the difference with Theorem 3.2, due to the fact that we are now near a nontrivial equilibrium branch.

Theorem 4.1. Consider the initial value problems

$$
\begin{aligned}
\frac{d x}{d \tau}= & \frac{\sqrt{\varepsilon}}{F^{\frac{3}{4}}} f(x, \tau) f_{1}\left(r_{1}\right)+\frac{\varepsilon}{F^{\frac{3}{2}}} f(x, \tau) g_{1}\left(r_{1}\right) \\
& +\frac{\varepsilon^{2}}{F} h(x, \tau) h_{1}\left(r_{1}\right)+O\left(\varepsilon^{2} F^{-3}\right), \quad x\left(\tau_{0}\right)=x_{0},
\end{aligned}
$$

and

$$
\begin{aligned}
\frac{d x_{a}}{d \tau}= & \frac{\varepsilon}{F^{\frac{3}{2}}} f^{10}\left(x_{a}, \tau\right) f_{1}\left(r_{1}\right)+\frac{\varepsilon}{F^{\frac{3}{2}}} g^{\circ}\left(x_{a}, \tau\right) g_{1}\left(r_{1}\right) \\
& +\frac{\varepsilon^{2}}{F} h^{\circ}\left(x_{a}, \tau\right) h_{1}\left(r_{1}\right), \quad x_{a}\left(\tau_{0}\right)=x_{0}
\end{aligned}
$$

with $f, g, h: \mathbb{R}^{2} \times \mathbb{R} \rightarrow \mathbb{R}^{2}, \tau \in\left[\delta_{1}^{*-1}(\varepsilon), \delta_{2}^{*-1}(\varepsilon)\right]$ with $\delta_{1}^{*}(\varepsilon)=o(1)$ and $\delta_{2}^{*}(\varepsilon)=O(\varepsilon)$ so that $F \in\left[\delta_{1}(\varepsilon), \delta_{2}(\varepsilon)\right]$ with $\varepsilon^{2 / 3}=o\left(\delta_{1}(\varepsilon)\right), \delta_{2}(\varepsilon)=O(1)$, and $\varepsilon \in\left(O, \varepsilon_{0}\right]$. 
Furthermore,

$$
f^{1}(x, \tau)=\nabla f(x, \tau) u^{1}(x, \tau) f_{1}\left(r_{1}\right)
$$

and

$$
u^{1}(x, \tau)=\int_{\tau_{0}}^{\tau} f(x, s) d s-\frac{1}{T} \int_{\tau_{0}}^{\tau_{0}+T} \int_{\tau_{0}}^{\tau} f(x, s) d s d \tau
$$

Suppose that

a) $f$ has a Lipschitz-continuous first derivative in $x$ and the vector functions $f_{1}$, $g_{1}, h_{1}, g, F^{1 / 2} h, \nabla g, \nabla F^{1 / 2} h, \nabla f$, and $\nabla^{2} f$ are continuous in the variables and bounded by a constant $M$, independent of $\varepsilon$, for $\tau \in\left[\delta_{1}^{*^{-1}}, \delta_{2}^{*^{-}}(\varepsilon)\right]$;

b) $f, g$, and $h$ are $T$-periodic in $\tau$, averages $f^{\circ}, g^{\circ}$, and $h^{\circ}\left(f^{1}\right.$ has average $\left.f^{10}\right)$. Moreover $f^{\circ}=0$.

Then $x(\tau)=x_{a}(\tau)+o(1)$ for $\tau \in\left[{\delta_{1}^{*}-1}^{-1}(\varepsilon), \delta_{2}^{*^{-1}}(\varepsilon)\right]$ or $F \in\left[\delta_{1}(\varepsilon), \delta_{2}(\varepsilon)\right]$.

Remark. The $O\left(\varepsilon^{1 / 2} F^{-3 / 4}\right)$-term did not appear in $\S 3$. Since $f^{\circ}=0$, we now have to apply second-order averaging (see also Sanders and Verhulst (1985)).

Proof. Define $y(\tau)$ by

$$
x(\tau)=y(\tau)+\varepsilon^{\frac{1}{2}} F^{-\frac{3}{4}} u^{1}(y(\tau), \tau) f_{1}\left(r_{1}\right)
$$

with

$$
u^{1}(y(\tau), \tau)=\int_{0}^{\tau} f(y, s) d s-\frac{1}{T} \int_{0}^{T} \int_{0}^{\tau} f(y, s) d s d \tau .
$$

$(f, g$, and $h$ are $T$-periodic in $\tau$.) Substitution in the differential equation (4.18) produces for $y$

$$
\begin{aligned}
\frac{d y}{d \tau}= & \left(\frac{\sqrt{\varepsilon} f^{\frac{1}{2}}\left(r_{1}\right)}{F^{\frac{3}{4}}}\right)^{2}\left(\nabla f(y, \tau) u^{1}(y, \tau) f_{1}\left(r_{1}\right)+g(y, \tau) g_{1}\left(r_{1}\right)+\varepsilon F^{\frac{1}{2}} h(y, \tau) h_{1}\left(r_{1}\right)\right) \\
& +O\left(e^{\frac{3}{2}} F^{-\frac{9}{4}}\right)
\end{aligned}
$$

We make the following estimate:

$$
\left\|x(\tau)-x_{a}(\tau)\right\| \leq\|x(\tau)-y(\tau)\|+\left\|y(\tau)-x_{a}(\tau)\right\| .
$$

For $\tau \in\left[\delta_{1}^{*-1}(\varepsilon), \delta_{2}^{*-1}(\varepsilon)\right], u^{1}(y(\tau), \tau)$ is bounded so that

$$
\|x(\tau)-y(\tau)\|=O\left(\varepsilon^{\frac{1}{2}} F^{-\frac{3}{4}}\right)=o(1) .
$$

We estimate $\left\|y(\tau)-x_{a}(\tau)\right\|$ in the same way as $\left\|z_{2}(\tau)-x_{2 a}(\tau)\right\|$ in Theorem 3.2 and obtain for $\tau \in\left[\delta_{1}^{*-1}(\varepsilon), \delta_{2}^{*-1}(\varepsilon)\right]$

$$
\left\|y(\tau)-x_{a}(\tau)\right\|=O\left(\varepsilon^{\frac{1}{2}} F^{-\frac{3}{4}}\right)=o(1) .
$$

This completes the proof of the theorem.

Corollary 4.2. For $0<\delta_{1}(\varepsilon) \leq F \leq F_{1}$ with $\varepsilon^{2 / 3}=o\left(\delta_{1}(\varepsilon)\right)$ and $F_{1}=O(1)-$ and thus for $\delta_{3}(\varepsilon) \leq \omega_{1}(F) \leq \delta_{4}(\varepsilon)$ with $\varepsilon^{1 / 3}=o\left(\delta_{3}(\varepsilon)\right.$ ) and $\delta_{4}(\varepsilon)=O(1)$ (see $(4.15 \mathrm{~b})$ ) - the solution of (1.2) has the following expansion:

$$
x(t)=\sqrt{\frac{F}{2}}+\sqrt{\varepsilon} r_{a}^{*} e^{\frac{-\kappa \varepsilon t}{2}} \omega_{1}^{-\frac{1}{2}}(F) \cos \left(\tau+\psi_{a}^{*}\right)+o\left(\sqrt{\varepsilon} \omega_{1}^{-\frac{1}{2}}(F)\right),
$$


with $\left(r_{a}^{*}, \psi_{a}^{*}\right)$ the solution of the system

$$
\frac{d r_{a}^{*}}{d \tau}=0, \quad r_{a}^{*}\left(t_{1}\right)=r_{0}^{*}
$$

$$
\frac{d \psi_{a}^{*}}{d \tau}=-\varepsilon\left(\frac{\varepsilon \kappa^{2}}{16 F}+\frac{3 e^{-\kappa \varepsilon t} r_{a}^{*^{2}}}{4 \cdot(2 F)^{\frac{3}{2}}}-\frac{15 e^{-\kappa \varepsilon t} r_{a}^{*^{2}}}{4 \cdot(2 F)^{\frac{3}{2}}}\right), \quad \psi_{a}^{*}\left(t_{1}\right)=\varphi_{0}^{*}
$$

Proof. Because (4.13c), (4.13d) is a special case of the initial value problem stated in Theorem 4.1, the averaged system (4.29) is an $o(1)$-approximation of the complete system (4.13c), (4.13d) for $\tau \in\left[\delta_{1}^{*-1}(\varepsilon), \delta_{2}^{*-1}(\varepsilon)\right]$ with $\delta_{1}^{*}(\varepsilon)=o(1)$ and $\delta_{2}^{*}(\varepsilon)=O(\varepsilon)$. It follows that for the solution of (1.2) approximation (4.28) holds when $0<\delta_{1}(\varepsilon) \leq F \leq F_{1}$ with $\varepsilon^{2 / 3}=o\left(\delta_{1}(\varepsilon)\right)$ and $F_{1}=O(1)$. In fact, from (4.13b) it follows that

$$
\tau=\left(\frac{-1}{3}(2 F)^{\frac{3}{2}}+\frac{1}{3}\left(2 F_{1}\right)^{\frac{3}{2}}\right) \varepsilon^{-1}
$$

On account of the symmetry of the problem we can also obtain a "slowly varying equilibrium solution" $x_{-l s v}(F)$ for $F>F_{c}$ by perturbing the dependent variable $x$ at $x_{-1}(F)=-\sqrt{ }(F / 2)$. By reflecting (4.28) with respect to the $z$-axis an asymptotic expansion of the solution of (1.2) in the neighbourhood of $x_{-l s v}(F)$ is obtained valid for $t \in\left[t_{c}+\delta_{5}^{-} 1(\varepsilon), t_{c}+\delta_{6}^{-1}(\varepsilon)\right]$ with $t_{c}=-F_{0} \varepsilon^{-1}, \delta_{5}(\varepsilon)=o\left(\varepsilon^{1 / 3}\right)$, and $\delta_{6}(\varepsilon)=O(\varepsilon)$. For $F=F_{c}=0$ the stable reduced solutions $x_{ \pm 1}(F)$ and the unstable one, $x_{0}(F)$, coalesce. Then approximation (4.28) does not hold anymore. It is remarked that then the angular velocity $\omega_{1}(F)$ tends to zero.

Finally, we remark that in the case without damping $(\kappa=0),(4.7 \mathrm{a})$ is a Hamiltonian system. Bosley and Kevorkian (1992) consider transient resonance in very slowly varying oscillatory Hamiltonian systems for which the leading-order frequency of the reduced system makes a continuous slow passage through zero. After the transformations $v \doteq r \cos \varphi, d v / d t=-\omega_{1}(F) r \sin \varphi$, and $p=r^{2} \omega_{1}(F)$ we obtain from (4.7)

$$
\begin{aligned}
& \frac{d p}{d \tau}=\sqrt{\varepsilon}\left(12 p^{\frac{3}{2}} \omega_{1}^{-\frac{3}{2}}(\varepsilon t) x_{1 s v}(\varepsilon t) \cos ^{2} \varphi \sin \varphi\right) \\
& +\varepsilon\left(4 p^{2} \omega_{1}^{-2}(\varepsilon t) \cos ^{3} \varphi \sin \varphi-2 p \omega_{1}^{-1}(\varepsilon t) \frac{d \omega_{1}}{d(\varepsilon t)} \sin ^{2} \varphi+p \omega_{1}^{-1} \frac{d \omega_{1}}{d(\varepsilon t)}\right) \\
& \frac{d \varphi}{d t}=\omega_{1}(\varepsilon t)+\sqrt{\varepsilon}\left(6 p^{\frac{1}{2}} \omega_{1}^{-\frac{3}{2}}(\varepsilon t) x_{1 s v}(\varepsilon t) \cos ^{3} \varphi\right) \\
& +\varepsilon\left(2 p \omega_{1}^{-2}(\varepsilon t) \cos ^{4} \varphi-\omega_{1}^{-1}(\varepsilon t) \frac{d \omega_{1}}{d(\varepsilon t)} \cos \varphi \sin \varphi\right)
\end{aligned}
$$

which is a Hamiltonian system with a Hamiltonian of the following form:

$$
\begin{aligned}
H= & \omega_{1}(\varepsilon t) p+\sqrt{\varepsilon}\left(3 p^{\frac{3}{2}} \omega_{1}^{-\frac{3}{2}}(\varepsilon t) x_{1 s v}(\varepsilon t) \cos \varphi+p^{\frac{3}{2}} \omega_{1}^{-\frac{3}{2}}(\varepsilon t) x_{1 s v}(\varepsilon t) \cos 3 \varphi\right) \\
& +\varepsilon\left(\frac{3}{8} p^{2} \omega_{1}^{-2}(\varepsilon t)+\frac{1}{2} p^{2} \omega_{1}^{-2}(\varepsilon t) \cos 2 \varphi+\frac{1}{8} p^{2} \omega_{1}^{-2}(\varepsilon t) \cos 4 \varphi\right)
\end{aligned}
$$


5. The transition layer equation and matching conditions. In order to obtain matching conditions for the local asymptotic solution describing the pitchfork bifurcation we determine the asymptotic development of $x$ when $F$ is in the neighbourhood of $F_{c}=0$. Near $F=0$ the reduced solutions $x_{0}(F)$ and $x_{ \pm 1}(F)$ are close to zero. Setting

$$
t=\frac{-F_{0}}{\varepsilon}+\varepsilon^{v-1} z \quad \text { or } F=\varepsilon^{v} z
$$

we obtain the following approximations for the $F$-dependent terms in the asymptotic expansions (3.31) and (4.28):

$$
\begin{gathered}
r_{1}=r_{1}^{*}=e^{\frac{1}{2} \kappa F_{0}}+\cdots \\
\omega_{0}^{2}(F)=-z \varepsilon^{v} \\
x_{1 s v}(F)=\left(\frac{z}{2}\right)^{\frac{1}{2}} \varepsilon^{\frac{1}{2} v}+\frac{1}{64}\left(\frac{z}{2}\right)^{-\frac{5}{2}} \varepsilon^{2-\frac{5}{2} v}+\cdots \\
+\frac{\kappa}{16}\left(\frac{z}{2}\right)^{-\frac{3}{2}} \varepsilon^{2-\frac{3}{2} v}+\cdots \\
\omega_{1}^{2}(F)=2 z \varepsilon^{v}+\frac{3}{16}\left(\frac{z}{2}\right)^{-2} \varepsilon^{2-2 v}+\cdots, \\
r_{a}^{*}=r_{0}^{*}, \quad r_{a}=r_{0}, \\
\varphi_{a}=\frac{2}{3}(-z)^{\frac{3}{2}} \varepsilon^{\frac{3}{2} v-1}+\frac{3}{4} r_{0}^{2} e^{\kappa F_{0}}(\ln (-z)+v \ln \varepsilon)+\psi_{0}+\cdots \\
\varphi_{a}^{*}=\frac{2 \sqrt{2}}{3}(z)^{\frac{3}{2}} \varepsilon^{\frac{3}{2} v-1}-\frac{3}{2} r_{0}^{*^{2}} e^{k F_{0}}(\ln (z)+v \ln \varepsilon)+\psi_{0}^{*}+\cdots
\end{gathered}
$$

where $\psi_{0}$ is a constant determined by the initial conditions. Consequently, near the bifurcation point the outer solutions (3.31) and (4.28) behave asymptotically as

$$
\begin{aligned}
x=r_{0} e^{\frac{\kappa F_{0}}{2}}(-z)^{-\frac{1}{4}} \varepsilon^{\frac{1}{2}-\frac{1}{4} v} & \times \sin \left\{\frac{2}{3}(-z)^{\frac{3}{2}} \varepsilon^{\frac{3}{2} v-1}+\frac{3}{4} r_{0}^{2} e^{\kappa F_{0}}(\ln (-z)+v \ln \varepsilon)+\psi_{0}+\cdots\right\}+\cdots \\
& \text { if } z \rightarrow-\infty \\
x= & \left(\frac{z}{2}\right) \varepsilon^{\frac{v}{2}}+\frac{1}{64}\left(\frac{z}{2}\right)^{-\frac{5}{2}} \varepsilon^{2-\frac{5}{2} v}+\cdots+\frac{\kappa}{16}\left(\frac{z}{2}\right)^{-\frac{3}{2}} \varepsilon^{2-\frac{3}{2} v}+\cdots \\
& +r_{0}^{*} e^{\frac{\kappa F_{0}}{2}}\left(2 z \varepsilon^{v}+\frac{3}{16}\left(\frac{z}{2}\right)^{-2} \varepsilon^{2-2 v}+\cdots\right)^{-\frac{1}{4}} \varepsilon^{\frac{1}{2}} \\
& \times \cos \left\{\frac{2 \sqrt{2}}{3}(z)^{\frac{3}{2}} \varepsilon^{\frac{3}{3} v-1}-\frac{3}{2} r_{0}^{*^{2}} e^{\kappa F_{0}}(\ln (z)+v \ln \varepsilon)+\psi_{0}^{*}+\cdots\right\}+\cdots \\
& \text { if } z \rightarrow \infty,
\end{aligned}
$$


with the dots standing for higher-order $\varepsilon$-terms or terms that are $o\left(|z|^{-1 / 4}\right)$. It is remarked that, in contrast with Haberman (1979), the terms $-3 r_{1}^{*^{2}} r^{2} \omega^{-3}(-F)$ and $-15 r_{1}^{*^{2}} r^{* 2} x_{1 s v}^{2} \omega_{1}^{-3}(F)$ in the integral of (4.13d) are equally important as $\omega_{1}(F) \rightarrow 0$. As we will see, both terms contribute to the matching condition for the pitchfork bifurcation, because both terms contribute to the logarithmic terms in (5.3b).

From the expansion (5.3) it is seen that the outer expansion breaks down if $v=2 / 3$. It implies that the transition layer (inner) equation follows from the scaling

$$
x=\varepsilon^{\frac{1}{3}} y(z)
$$

Another way to obtain this equation is based on the analysis of significant degenerations of the differential equation. We then put

$$
F=\varepsilon^{v} z, \quad x=\varepsilon^{\mu} y(z) .
$$

There is a significant degeneration for $\mu=1 / 3, v=2 / 3$ of the following form:

$$
\frac{d^{2} y}{d z^{2}}=y z-2 y^{3} \text {. }
$$

This equation, a nonlinear extension of the Airy equation, also occurs in a number of problems of quantum field theory and in the theory of nonlinear evolution equations. It is the second Painlevé equation, being one of the six canonical Painlevé equations of the form

$$
\frac{d^{2} y}{d z^{2}}=R\left(z, y, \frac{d y}{d z}\right)
$$

where $R$ is rational in $y$ and $d y / d z$ and analytic in $z$. The first integral of these Painlevé equations has no nonstationary critical points (the Painlevé property) (see Painlevé (1900)) and cannot be reduced to linear equations by local transformations (i.e., their first integral cannot be expressed in terms of known special functions; see also Levi and Winternitz (1991)).

The correct scaling for the transition layer equation also follows from the time region, where according to Theorems 3.2 and 4.1 the outer solutions (3.31) and (4.28) are not valid. In $\S \S 3$ and 4 we have seen that the averaging procedures cannot be applied anymore if $F=O\left(\varepsilon^{2 / 3}\right)$ (or $t=-F_{0} \varepsilon^{-1}+O\left(\varepsilon^{-1 / 3}\right)$ ). In this region a new local approximation, satisfying the significant degeneration (5.6), is constructed, and its validity is proved. Its validity domain may be extended backward and forward, overlapping the other (outer) approximations, so that integration constants follow from matching with these approximations.

We will first prove that the transition layer solution is a local approximation of the solution of the complete equation. For that purpose we will first prove the following lemma.

LEMMA 5.1. The initial value problem

$$
\frac{d^{2} y_{p}}{d z^{2}}=y_{p} z-2 y_{p}^{3}, \quad y_{p}(-M)=a, \quad \frac{d y_{p}}{d z}(-M)=b
$$

with $M>0$ arbitrarily large, has a bounded real solution for $-M \leq z \leq M$ for any $M>0$. 
Proof. From (5.8) it follows that

$$
\left(\frac{d y_{p}}{d z}\right)^{2}=z y_{p}^{2}-\int y_{p}^{2}+C_{1}-y_{p}^{4}
$$

with $C_{1}$ a constant. Certainly, $\left(d y_{p} / d z\right)^{2} \leq M y_{p}^{2}+C_{2}$, with $C_{2}$ a positive constant. Thus,

$$
\frac{d y_{p}}{d z} \leq \sqrt{M y_{p}^{2}+C_{2}}
$$

So $\int\left(y_{p}^{2}+C_{3}\right)^{-1 / 2} d y_{p} \leq C_{4}$ with $C_{3} \geq 0$ and $C_{4} \geq 0$. Finally, we obtain

$$
\log \left|y_{p}+\sqrt{y_{p}^{2}+C_{3}}\right| \leq C_{4}
$$

Thus, $y_{p}$ and $d y_{p} / d z$ are bounded.

In fact, it is known (see, e.g., Levi and Winternitz (1991)) that the only singularities that any solution of Painlevé II can have for finite $z$ are poles. However, poles cannot occur, as a behaviour of the type $y_{p}(z) \sim c\left(z-z_{0}\right)^{n}, c \in \mathbb{R}, n \in \mathbf{Z}$, is excluded in (5.6) because of the sign of the nonlinear term. We can now prove the following approximation theorem.

THEOREM 5.2. Consider the system

$$
\left(\begin{array}{l}
\frac{d x}{d z} \\
\frac{d y}{d z}
\end{array}\right)=\left(\begin{array}{c}
y \\
z x-x^{3}
\end{array}\right)+\varepsilon^{\frac{1}{3}}\left(\begin{array}{c}
0 \\
a z^{2}+b z
\end{array}\right)+\varepsilon^{\frac{2}{3}}\left(\begin{array}{c}
0 \\
y+c z
\end{array}\right)+\varepsilon\left(\begin{array}{c}
0 \\
d z^{3}
\end{array}\right)
$$

or

$$
\frac{d u}{d z}=f_{0}(z, u)+\varepsilon^{\frac{1}{3}} R(z, u, \varepsilon)
$$

with $u(-M)=\eta, z \in[-M, M], 0<\varepsilon \leq \varepsilon_{0}$, and $u$ a two-dimensional vector function. Assume that

a) $R(z, u, \varepsilon)$ is continuous in $z, u$, and $\varepsilon$ and Lipschitz-continuous in $u$.

b) $f_{0}(z, u)$ is continuous in $z$ and $u$ and continuously differentiable in $z$. Let $u_{p}=\left(x_{p}, y_{p}\right)$ be the solution of

$$
\frac{d u_{p}}{d z}=f_{0}\left(z, u_{p}\right), \quad u_{p}(-M)=\eta .
$$

Then $\left\|u(z)-u_{p}(z)\right\| \leq k \varepsilon^{1 / 3}, k$ a constant, for $|z|<M$.

Proof. From (5.13) and (5.14) it follows that

$$
\begin{aligned}
u(z)-u_{p}(z)= & \int_{-M}^{z}\left[f_{0}(\tau, u(\tau))-f_{0}\left(\tau, u_{p}(\tau)\right)\right] d \tau+\varepsilon^{\frac{1}{3}} \\
& \times \int_{-M}^{z} R(\tau, u(\tau), \varepsilon) d \tau .
\end{aligned}
$$

We have

$$
\begin{aligned}
\| R(\tau, u(\tau), \varepsilon \| & \leq\left\|R(\tau, u(\tau), \varepsilon)-R\left(\tau, u_{p}(\tau), \varepsilon\right)\right\|+\left\|R\left(\tau, u_{p}(\tau), \varepsilon\right)\right\| \\
& \leq L_{1} \cdot\left\|u(\tau)-u_{p}(\tau)\right\|+M_{1}
\end{aligned}
$$


because of the Lipschitz-continuity of $R$, the boundedness of $u_{p}$ (see Lemma 5.1), and $z \in[-M, M]$. Using the Lipschitz-continuity of $f_{0}$ we now obtain from (5.15) and $(5.16)$

$$
\begin{aligned}
\left\|u(z)-u_{p}(z)\right\| \leq & \int_{-M}^{z} L \cdot\left\|u(\tau)-u_{p}(\tau)\right\| d \tau \\
& +\varepsilon^{\frac{1}{3}} \int_{-M}^{z}\left[L_{1} \cdot\left\|u(\tau)-u_{p}(\tau)\right\|+M_{1}\right] d \tau .
\end{aligned}
$$

Application of the lemma of Gronwall yields the inequality

$$
\left\|u(z)-u_{p}(z)\right\| \leq \varepsilon^{\frac{1}{3}} \frac{M_{1}}{L+\varepsilon^{\frac{1}{3}} L_{1}} e^{L(z+M)}-\varepsilon^{\frac{1}{3}} \frac{M_{1}}{L+\varepsilon^{\frac{1}{3}} L_{1}} .
$$

So it follows that $u(z)-u_{p}(z)=O\left(\varepsilon^{1 / 3}\right)$ on the time-scale 1. From this it follows that we are allowed to say

$$
u(z)=u_{p}(z)+\varepsilon^{\frac{1}{3}} \varphi(z, \varepsilon) .
$$

By using the same techniques, integral equations, and the lemma of Gronwall, we can also show that substitution of

$$
u^{*}(z)=u_{p}(z)+\varepsilon^{\frac{1}{3}} u_{1}(z)+\varepsilon^{\frac{2}{3}} u_{2}(z)+\varepsilon u_{3}(z)
$$

yields an $O\left(\varepsilon^{4 / 3}\right)$-approximation for the solution of (5.13) for $|z|<M$.

From Theorem 5.2 this corollary immediately follows.

COROLLARY 5.3. The solution of the significant degeneration (5.6) is an $O\left(\varepsilon^{\frac{1}{3}}\right)$ approximation of the solution (1.2) for $t \in\left[-F_{0} \varepsilon^{-1}-M \varepsilon^{-1 / 3},-F_{0} \varepsilon^{-1}+M \varepsilon^{-1 / 3}\right]$ or $F \in\left[-M \varepsilon^{2 / 3}, M \varepsilon^{2 / 3}\right]$ with $M$ an arbitrary positive constant.

The extension theorem (Eckhaus, 1979) states that (when $\varepsilon$ gets smaller) we may extend the interval during which the approximations are valid, possibly at the cost of accuracy. We now give the precise formulation of this theorem.

THEOREM 5.4 (extension theorem). Let, for $-M \leq z \leq M$ with $M$ arbitrary but fixed and $\varepsilon$-independent,

$$
\left|y_{1}(z, \varepsilon)-y_{2}(z, \varepsilon)\right|=o(1)
$$

Then order functions $\delta_{e}(\varepsilon), \delta_{1 e}(\varepsilon)=o(1)$ exist such that

$$
\left|y_{1}(z, \varepsilon)-y_{2}(z, \varepsilon)\right|=O\left(\delta_{1 e}\right)
$$

for $-\delta_{e}^{-1}(\varepsilon) \leq z \leq \delta_{e}^{-1}(\varepsilon)$.

For a discussion and proof of the extension theorem we refer to Eckhaus (1979). From the approximation theorem 5.2 and the extension theorem it follows that the domain of validity of the local Painlevé approximation can be extended forward and backward to $F \in\left[-\varepsilon^{2 / 3} \delta_{e}^{-1}(\varepsilon), \varepsilon^{2 / 3} \delta_{e}^{-1}(\varepsilon)\right]$ with $\delta_{e}(\varepsilon)=o(1)$. Thus overlap with the domains, where outer approximations are valid, is ensured and the integration constants can be matched.

At the time that a pitchfork bifurcation is expected, (5.6) holds. Its solution must match the outer solution as given by $(5.3 \mathrm{~b})$ with $v=2 / 3$ :

$$
\begin{aligned}
y \sim & \left(\frac{z}{2}\right)^{\frac{1}{2}}+r_{0}^{*} e^{\frac{\kappa F_{0}}{2}}(2 z)^{-\frac{1}{4}} \cos \left\{\frac{1}{3}(2 z)^{\frac{3}{2}}-\frac{3}{2} r_{0}^{*^{2}} e^{\kappa F_{0}} \ln (z)+\zeta_{0}^{*}\right\} \\
& +o\left(z^{-\frac{1}{4}}\right) \text { as } z \rightarrow \infty
\end{aligned}
$$


with $\xi_{0}^{*}$ depending on the initial conditions and $\ln (\varepsilon)$. This is the parabolic matching condition after passage of the bifurcation point when the solution remains near the stable outer solution $x=x_{1}$. When the solution remains near the other stable outer equilibrium solution $x=x_{-1}$ this matching condition is obtained by reflecting (5.23) with respect to the $z$-axis. The asymptotic condition is automatically fullfilled by (5.6).

5.1. Two special solutions of the second Painleve transcendent. When $r_{0}^{*}=0$ we obtain a specific solution of the Painleve transcendent: the one that matches (5.3b) with $r_{0}^{*}=0$ and that reflects the asymptotic behaviour of the slowly varying equilibrium solution $x_{1 s v}(F)$ when the transition layer is approached. It is noted that (5.3b) is then independent of $\varepsilon$ (and $\left.\psi_{0}^{*}\right)$. For $z \gg 1$, this solution has an asymptotic series of the form

$$
y(z)=\sum_{n=1}^{\infty} c_{n}\left(\frac{z}{2}\right)^{\frac{1}{2}-3(n-1)},
$$

with $c_{n}$ satisfying a recurrence relation:

$$
c_{1}=1, \quad c_{2}=\frac{1}{64},
$$

and for $n \geq 3$

$$
\begin{aligned}
& \frac{1}{4} c_{n}\left(\frac{7}{2}-3 n\right)\left(\frac{5}{2}-3 n\right) \\
& =\left\{\begin{array}{l}
2 c_{n+1}-\sum_{i \geq 1, j \geq 1, i+2 j=n+3} 6 c_{i} c_{j}^{2}-2 c_{(n+3) / 3}, \quad n \equiv 0(\bmod 3), \\
2 n+1-c_{i \geq 1, j \geq 1, i+2 j=n+3} c_{j}^{2}, \quad n \not \equiv 0(\bmod 3) .
\end{array}\right.
\end{aligned}
$$

This solution is illustrated in Figure 4.

In the same way, the solution of (5.6) must match the outer solution as given by (5.3a) with $v=2 / 3$ :

$$
\begin{aligned}
y \sim & r_{0} e^{\frac{\kappa F_{0}}{2}}(-z)^{-\frac{1}{4}} \sin \left\{\frac{2}{3}(-z)^{\frac{3}{2}}+\frac{3}{4} r_{0}^{2} e^{\kappa F_{0}} \ln (-z)+\zeta_{0}\right\} \\
& +o\left((-z)^{\frac{1}{4}}\right) \text { as } z \rightarrow-\infty .
\end{aligned}
$$

Again, the asymptotic condition is automatically fulfilled by (5.6). In fact, the equation is satisfied by a series, which has the form (5.26) for $z \rightarrow-\infty$ :

$$
\begin{aligned}
y(z)=\sum_{n=1}^{\infty}\left\{a_{2 n-1,2 n-1} \sin \left((2 n-1)\left(\psi+\psi_{0}\right)\right)\right. \\
+\sum_{m=1}^{n-1}\left[b_{2 m-1,2 n-1} \sin \left((2 m-1)\left(\psi+\psi_{0}\right)\right)\right. \\
\left.\left.\quad+c_{2 m-1,2 n-1} \cos \left((2 m-1)\left(\psi+\psi_{0}\right)\right)\right]\right\} \cdot(-z)^{-\frac{6 n+5}{4}}
\end{aligned}
$$

with

$$
\psi=\frac{2}{3}(-z)^{\frac{3}{2}}+\frac{3}{4} a_{1,1}^{2} \ln (-z) .
$$




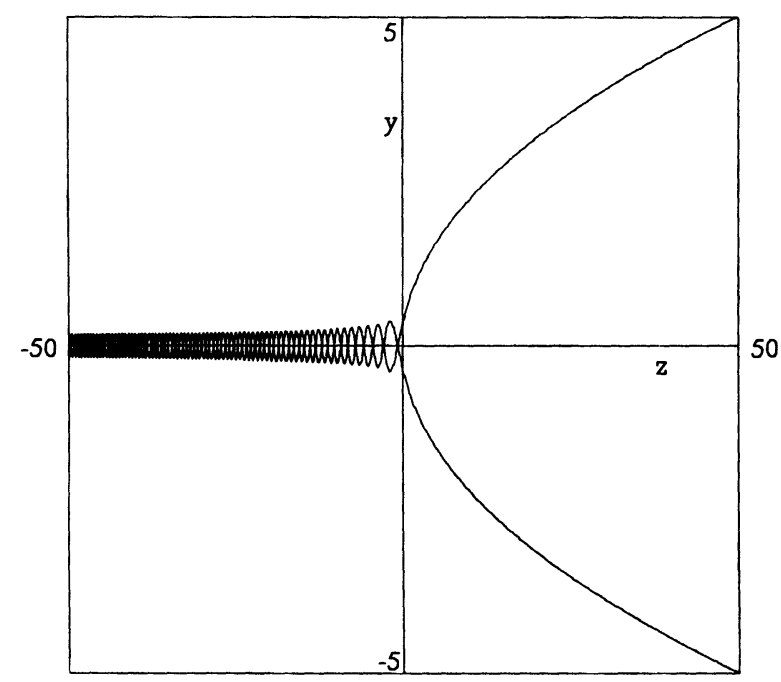

FIG. 4. Numerical approximation of the solutions of (5.6) that match (5.3b) with $r_{0}^{*}=0$ or match the reflection of $(5.3 \mathrm{~b})$ with respect to the $z$-axis with $r_{0}^{*}=0$.

The two arbitrary parameters are $a_{1,1}$ and $\psi_{0}$; the other parameters can be obtained from $a_{1,1}$ by a recurrent relation. In Figure 5 we illustrate the various analytical approximations of $\S \S 3,4$, and 5 with their domain of validity. As we have shown, the inner and outer approximations overlap. In the next section we will show the connection between the parameters $\left(r_{0} e^{-\kappa F_{0} / 2}, \zeta_{0}\right)$ and $\left(r_{0}^{*} e^{-\kappa F_{0} / 2}, \zeta_{0}^{*}\right)$.

6. The prediction of the behaviour of solutions after passing the bifurcation point at the basis of the initial values. If the state of a mechanical system at a certain moment is known, we wish to predict its future behaviour. As we have illustrated in Figure 6, most solutions of (1.2) will grow polynomially after passing the pitchfork bifurcation point. From the initial values of the original system (1.2) with $F(0)=F_{0}<F_{c}=0$, it will be deduced which of the two stable branches will be followed after passage of the bifurcation point.

In $\S 5$ we derived the following asymptotic behaviour of $y(z)$ satisfying $d^{2} y / d z^{2}=$ $y z-2 y^{3}$ for $z \ll-1$ :

$$
y(z)=\gamma(-z)^{-\frac{1}{4}} \sin \left\{\frac{2}{3}(-z)^{\frac{3}{2}}+\frac{3}{4} \gamma^{2} \ln (-z)+\zeta_{0}\right\}+o\left((-z)^{-1 / 4}\right)
$$

where

$$
F_{0}<F_{c}=0, \quad \omega_{0}(F)=\sqrt{-F}
$$

$$
r_{0}=\sqrt{\frac{\omega_{0}\left(F_{0}\right) x^{2}(0)}{\varepsilon}+\frac{\left(\frac{d x}{d t}(0)\right)^{2}}{\varepsilon \omega_{0}\left(F_{0}\right)}}
$$




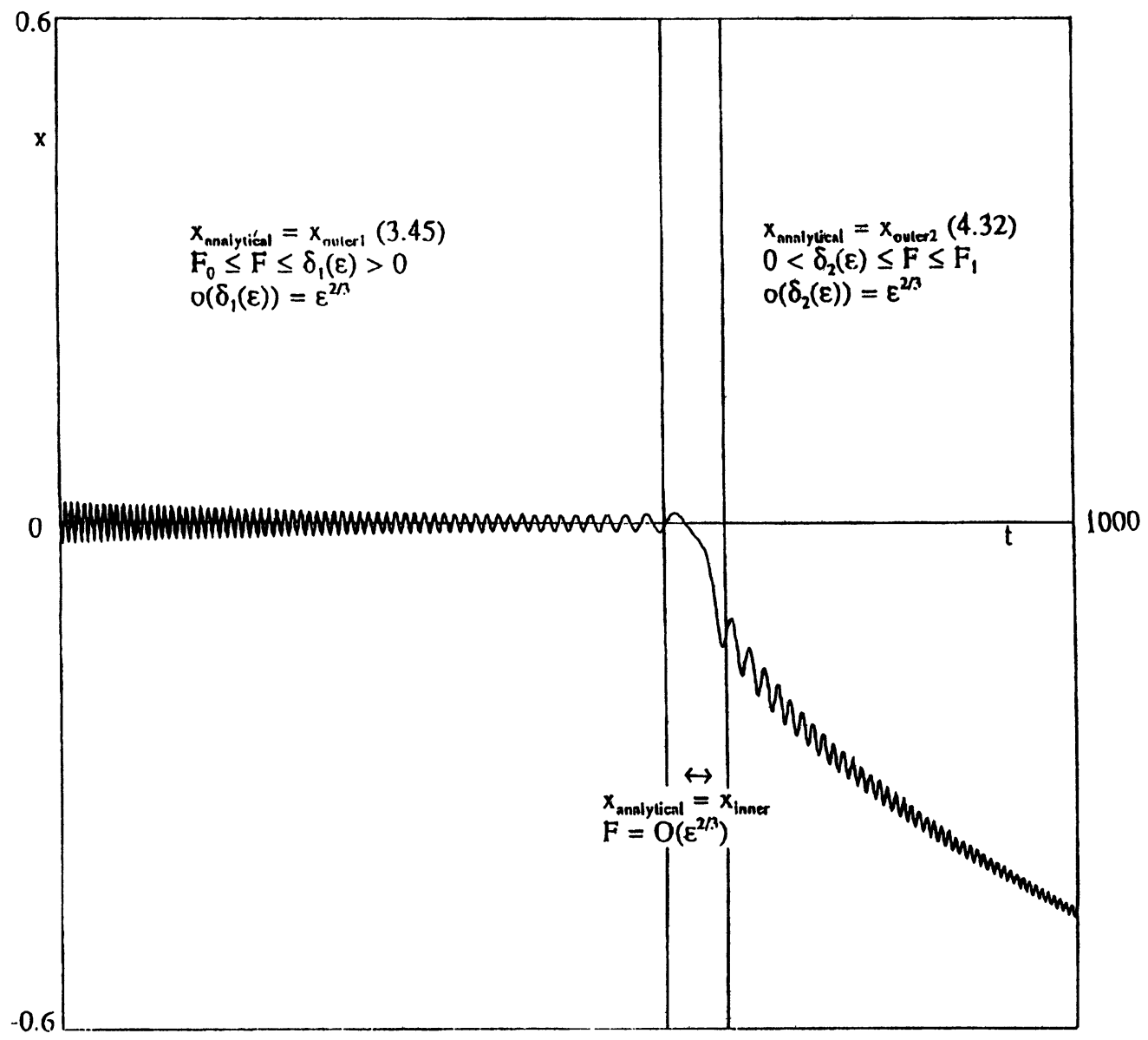

FIG. 5. The composite analytical solution: $x_{\text {analytical }}=x_{\text {outer }}+x_{\text {inner }}-x_{m a t c h}$ for (1.2) with $x(0)=0.04, x^{\prime}(0)=0, F(0)=-1, k=0.005$, and $\varepsilon=0.0016$.

(6.1e) $\zeta_{0}=\frac{-2}{3 \varepsilon}\left(-F_{0}\right)^{\frac{3}{2}}-\frac{3}{4} r_{0}^{2} \ln \left|F_{0}\right|+\frac{1}{2} \gamma^{2} \ln \varepsilon+\frac{3}{4} \kappa e^{\kappa F_{0}} r_{0}^{2} \int_{F_{0}}^{0} e^{\kappa F} \ln |F| d F-\varphi_{0}+\pi$ and for $z \gg 1$

$$
y(z)= \pm \sqrt{\frac{z}{2}} \pm \beta(2 z)^{-\frac{1}{4}} \cos \left\{\frac{2 \sqrt{2}}{3} z^{\frac{3}{2}}-\frac{3}{2} \beta^{2} \ln (z)+\zeta_{0}^{*}\right\}+o\left(z^{-1 / 4}\right)
$$

where

$$
\beta=r_{0}^{*} e^{\frac{\kappa F_{0}}{2}}
$$

$$
F_{1}>F_{c}=0, \quad \omega_{1}(F) \approx \sqrt{2 F} \text { for } \varepsilon^{\frac{2}{3}}=o(F)
$$




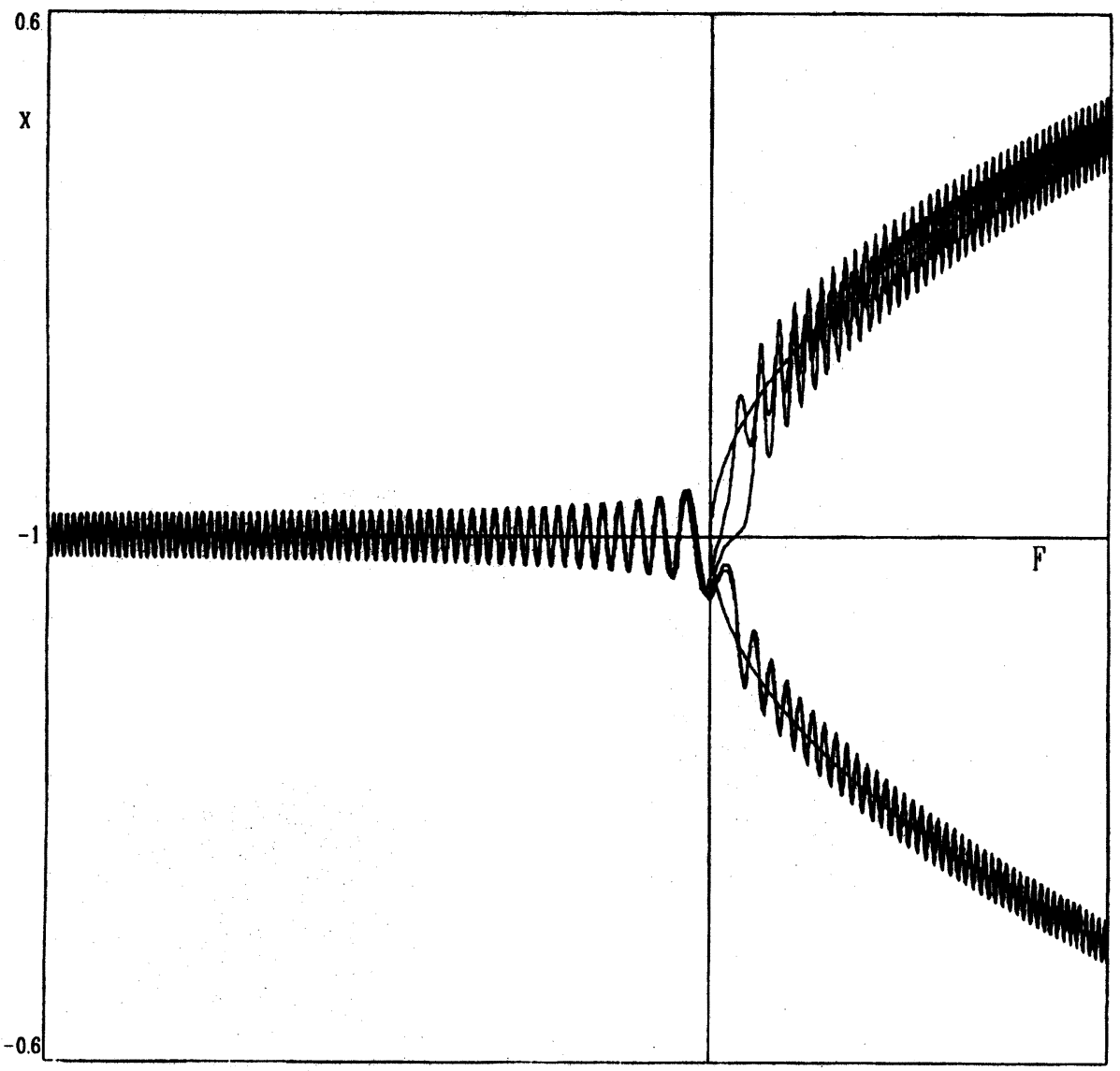

FIG. 6. Solutions of (1.2) for various sets of initial values with $F(0)=-1<0, \varepsilon=0.0025$, and $k=0$.

$$
r_{0}^{*}=\sqrt{\frac{\omega_{1}\left(F_{1}\right) x^{2}\left(t_{1}\right)}{\varepsilon}+\frac{\left(\frac{d x}{d t}\left(t_{1}\right)\right)^{2}}{\varepsilon \omega_{1}\left(F_{1}\right)}}
$$

$$
\zeta_{0}^{*}=\frac{-2 \sqrt{2}}{3 \varepsilon}\left(F_{1}\right)^{3 / 2}+\frac{3}{2} \beta^{2} e^{-\kappa F_{1}} \ln \left(F_{1}\right)-\beta^{2} \ln \varepsilon-\frac{3}{2} \kappa \beta^{2} \int_{F_{1}}^{0} e^{-\kappa F} \ln |F| d F+\varphi_{0}^{*}
$$

The properties of the real solutions of (5.6) follow from the properties of the general pure imaginary solutions of

$$
\frac{d^{2} u}{d s^{2}}-s u-2 u^{3}=0
$$

In Its, Fokas, and Kapaev (1994) an important result concerning this differential equation is stated: there is a connection between the behaviour of solutions for $s \rightarrow$ $-\infty$ with the behaviour for $s \rightarrow \infty$. Moreover, they discuss the connection between a method of Deift and Zhou (1993), who obtain a rigorous derivation of the connection 
problem for the second Painlevé equation, and an approach which was proposed by Kitaev (1989). We now give the formulation of the result.

THEOREM 6.1. Let $u(s)$ be an arbitrary solution of (6.3). Then the following assertions hold for $u(s)$ :

a) $u(s)$ is smooth for every $s \in \mathbb{R}$ and has the following asymptotics as $s \rightarrow-\infty$ :

$$
u(s)=i \alpha(-s)^{-\frac{1}{4}} \sin \left\{\frac{2}{3}(-s)^{\frac{3}{2}}+\frac{3}{4} \alpha^{2} \ln (-s)+\varphi\right\}+o\left((-s)^{-\frac{1}{4}}\right),
$$

where the numbers $\alpha>0$ and $0 \leq \varphi<2 \pi$ may be arbitrary and are parameters of the solution $u(s)$.

b) If the parameters $\alpha$ and $\varphi$ of the solution $u(s)$ are connected by the relation

$$
\varphi=\frac{3}{2} \alpha^{2} \ln 2-\frac{\pi}{4}-\arg \Gamma\left(i \cdot \frac{\alpha^{2}}{2}\right)+\varepsilon \pi(\bmod 2 \pi), \quad \varepsilon=0,1,
$$

then as $s \rightarrow+\infty$ the solution $u(s)$ decreases exponentially:

$$
u(s)=\frac{i a}{2 \sqrt{\pi}} s^{-\frac{1}{4}} e^{-2 s^{3 / 2}}(1+o(1))
$$

where $a^{2}=\exp \left(\pi \alpha^{2}\right)-1$ and $\operatorname{sgn} a=2\left(\frac{1}{2}-\varepsilon\right)$.

c) If (6.5) fails to hold (general position), then as $s \rightarrow+\infty$ the solution $u(s)$ grows polynomially:

$$
u(s)= \pm i \cdot \frac{\sqrt{s}}{2} \pm i \cdot(2 s)^{-\frac{1}{4}} \rho \cos \left\{\frac{2 \sqrt{2}}{3} s^{\frac{3}{2}}-\frac{3}{2} \rho^{2} \ln s+\theta\right\}+o\left(s^{-\frac{1}{4}}\right) .
$$

d) In the asymptotics (6.7) all values of $\rho>0$ and $0 \leq \theta<2 \pi$ are possible; these quantities characterize the solution $u(s)$ uniquely. The parameters $\rho, \theta$, and the choice of the sign in (6.7) are explicitly determined from the parameters $\alpha$ and $\varphi$ :

$$
\rho^{2}=\frac{1}{\pi} \ln \frac{1+\left|p^{2}\right|}{2-|\operatorname{Im} p|}
$$

$$
\theta=-\frac{3 \pi}{4}-\frac{7}{2} \rho^{2} \ln 2+\arg \Gamma\left(i \rho^{2}\right)+\arg \left(1+p^{2}\right)
$$

where

$$
p=\left(e^{\pi \alpha^{2}}-1\right)^{\frac{1}{2}} \exp \left\{i \frac{3}{2} \alpha^{2} \ln 2-i \frac{\pi}{4}-i \arg \Gamma\left(\frac{i \alpha^{2}}{2}\right)-i \varphi\right\}
$$

and the upper sign in (6.7) is taken if $\operatorname{Im} p<0$.

For a proof of this theorem we refer to Its, Fokas, and Kapaev (1994). This result is important for the analysis of the large class of physical problems. It confirms the asymptotic results we obtained for the real solutions of (5.6). Moreover, it connects the integration constants in the asymptotic solution for $z \rightarrow-\infty$ with those in the one for $z \rightarrow+\infty$. Furthermore, "separating" solutions, which follow the unstable branch beyond the bifurcation point, are singled out. A proof of the completeness of the asymptotic description of the solution of the differential equation is given in Its, Fokas, and Kapaev (1994). In order to prove this theorem, the method of isomonodromy deformations as formulated by Flaschka and Newell (1980) has been used. 
This method, as well as Laplace's method in the linear theory, allows us to compute explicitly connection formulas for Painlevé equations. The essence of the isomonodromy method is that a Stokes multiplier of an associated system is the first integral of the nonlinear Painlevé equation (see also Its and Novokshenov (1986)).

With the aid of Theorem 6.1 and (6.1) we can describe all solutions of (1.2) depending on the initial values and the value of $\varepsilon$; we can predict the branch that is followed after passing the bifurcation point as well as the type of behaviour the solution exhibits. In terms of our system we have obtained in (6.5) an "angle-amplitude relation" for a solution of (1.2) that separates the solutions following the stable upper branch from the ones that take the stable lower branch after passage of the bifurcation point. The quantity $\varphi$, stated in (6.5), is discontinuous in $\alpha=0$. However, this is the case when we will always stay on the equilibrium solution $x=0$ of (1.2) that becomes unstable for $t>0$. For $\alpha$ small, the quantity $\varphi$ tends to $\pi / 4+\varepsilon \pi, \varepsilon=0,1$. In the case of a system with damping $(k \neq 0)$, the amplitude of the oscillation around zero is small. In that case, solutions will follow the stable upper branch when the phase $\zeta_{0}$ (see (6.1a)) is approximately in the interval $\langle\pi / 4+2 n \pi, 5 \pi / 4+2 n \pi\rangle, n \in \mathbb{N}$. In Figure 7 the separating solution has been sketched in the $\gamma, \zeta_{0}$-plane. Numerical experiments confirm these results. Furthermore, in Figure 8, we have illustrated in the phase plane of the original system (1.2) which branch solutions will follow after passing the bifurcation point for fixed $\varepsilon$ and $F_{0}$, depending on the initial values.

7. The general case of second-order pitchfork bifurcations with damping. We consider the class of mechanical problems that can be described by the second-order nonlinear differential equation

$$
\frac{d^{2} x}{d t^{2}}+k \cdot \frac{d x}{d t}=G(x, F)
$$

from which the parameter $F$ slowly varies in time: $F=F(\varepsilon t)$. The damping is assumed to be small: $k=\kappa \varepsilon$. Solution curves of (7.1) are defined on the time-scale

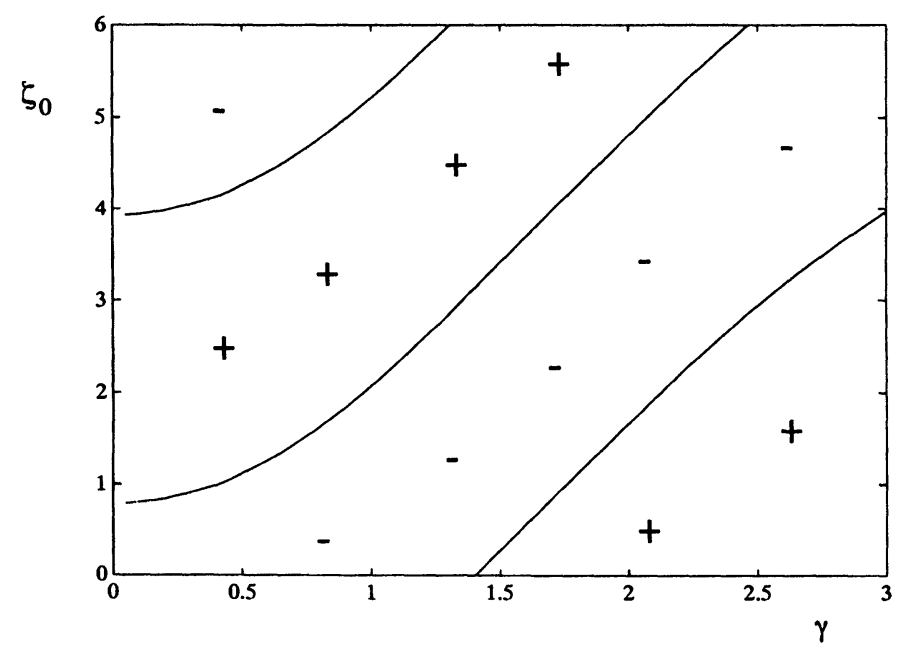

FIG. 7. The branch followed after passage of the bifurcation point depending on $\gamma$ and $\zeta_{0}$. + denotes the stable upper branch, and - the stable lower branch. In the separating case that is represented by the solid lines, solutions of (1.2) will approach the unstable branch beyond the bifurcation point. 


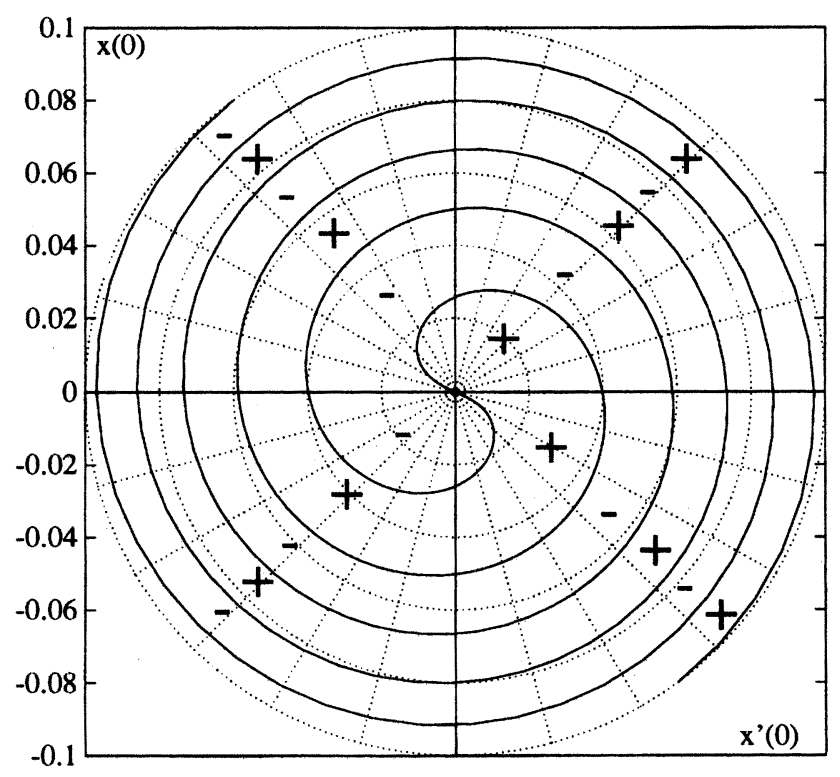

FIG. 8. The branch that will be approached sketched in the $x^{\prime}(0), x(0)$-plane of (1.2) for $\varepsilon=$ $0.0025, F_{0}=-1$, and $k=0$. The solid lines reflect approximations of the initial values for which the unstable branch will be approached after bifurcation. + denotes that the stable upper branch will be followed after bifurcation, and - denotes the stable lower branch.

$O(1 / \varepsilon)$ if the following condition is satisfied:

$$
\begin{aligned}
& \int_{-\frac{M}{\varepsilon}}^{\frac{M}{\varepsilon}} G(x, \varepsilon t) d t \leq M_{1} x^{2}+M_{2} \quad \text { with } \\
& \quad M, M_{1}, M_{2} \varepsilon \text {-independent positive constants. }
\end{aligned}
$$

For fixed $F$ the linear stability of an equilibrium solution $x_{E}(F)$ is determined by the linearization of (7.1). A critical value $F_{c}$ of $F$ occurs if

$$
\frac{\partial G}{\partial x}\left(x_{E}\left(F_{c}\right), F_{c}\right)=0
$$

While $\partial^{2} G / \partial x \partial F \neq 0$, this value separates stable from unstable solutions. In this study we also have that at the critical value

$$
\frac{\partial G}{\partial F}\left(x_{E}\left(F_{c}\right), F_{c}\right)=0 .
$$

In the neighbourhood of $x=x_{E}\left(F_{c}\right), F=F_{c}$ we assume $G$ to have the following form:

$$
\begin{aligned}
G(x, F)= & \alpha_{30}\left(\left(x-x_{E}\left(F_{c}\right)\right)-\beta_{1}\left(F-F_{c}\right)+\cdots\right) \\
& \times\left(\left(x-x_{E}\left(F_{c}\right)\right)^{2}-\sigma^{2}\left(F-F_{c}\right)+\cdots\right) \\
= & \alpha_{11}\left(x-x_{E}\left(F_{c}\right)\right)\left(F-F_{c}\right)+\alpha_{02}\left(F-F_{c}\right)^{2}+\alpha_{30}\left(x-x_{E}\left(F_{c}\right)\right)^{3} \\
& +\alpha_{21}\left(x-x_{E}\left(F_{c}\right)\right)^{2}\left(F-F_{c}\right)+\alpha_{12}\left(x-x_{E}\left(F_{c}\right)\right)\left(F-F_{c}\right)^{2} \\
& +\alpha_{03}\left(F-F_{c}\right)^{3}+\cdots
\end{aligned}
$$


where $\alpha_{n m}=\frac{1}{n ! m !}(\partial / \partial x)^{n}(\partial / \partial F)^{m} G\left(x_{E}\left(F_{c}\right), F_{c}\right)$.

This phenomenon has been called a pitchfork bifurcation. This case is characterized by $\alpha_{20}=0$. Note that $\alpha_{30}<0$ (so that $G(x, F)<0$ for $x$ sufficiently large) and $\alpha_{11}>0$ (so that a parabolic curve exists for $F>F_{c}$ ). In this study $\alpha_{21}=-\beta_{1} \alpha_{30}$ and $\alpha_{11}=-\sigma^{2} \alpha_{30}>0$. The moment of pitchfork bifurcation is approximated by $t_{c}$ satisfying

$$
F\left(\varepsilon t_{c}\right)=F_{c}
$$

In the same way as in $\S \S 3$ and 4 we can obtain an asymptotic expansion for a slowly varying equilibrium solution of (7.1) and consider $\sqrt{ } \varepsilon$-perturbations of this solution. We obtain a slowly varying oscillator approaching a turning point as the frequency $\omega(F)$ tends to zero for $F \rightarrow F_{c}$. We will see that two different cases must be analysed: the transition from the parabolic arc to the straight line curve as $F$ decreases through $F_{c}$ and the opposite case, which occurs as $F$ is increased through $F_{c}$. With the aid of averaging techniques we obtain asymptotic expansions of the solutions in the neighbourhood of the slowly varying equilibria. The expansions break down when $\varepsilon t=O\left(\varepsilon^{2 / 3}\right)$. Matching implies that the local inner equation follows from the scaling

$$
x=x_{E}\left(F_{c}\right)+\varepsilon^{\frac{1}{3}} y(z), \quad F=F_{c}+\varepsilon^{\frac{2}{3}} z .
$$

Making these scale changes we find that (7.1) transforms into

$$
\frac{d^{2} y}{d z^{2}}=\alpha_{11} F_{c}^{\prime} z y+\alpha_{30} y^{3}+O\left(\varepsilon^{\frac{1}{6}}\right)
$$

with

$$
F_{c}^{\prime}=\frac{d F}{d(\varepsilon t)}\left(\varepsilon t_{c}\right) .
$$

This equation must be solved with the matching conditions following from the asymptotic expansions for the outer solutions. We obtain the following asymptotic conditions: for $F \uparrow F_{c}$,

$$
\begin{gathered}
\omega^{2}(F) \rightarrow \sigma^{2} \alpha_{30}\left(F-F_{c}\right) F_{c}^{\prime} \\
w \sim\left(-\sigma^{2} \alpha_{30} F_{c}^{\prime}\right)^{-\frac{1}{4}} \rho_{0}(-z)^{-\frac{1}{4}} \sin \left\{\frac{2}{3}\left(\alpha_{11} F_{c}^{\prime}\right)^{\frac{1}{2}}(-z)^{\frac{3}{2}}+\frac{3 \rho_{0}^{2}}{8 \sigma^{2} F_{c}^{\prime}} \ln (-z)+\psi_{0}\right\} \\
\text { as } z \rightarrow-\infty
\end{gathered}
$$

and for $F \downarrow F_{c}$,

$$
\begin{gathered}
\omega^{2}(F) \rightarrow-2 \sigma^{2} \alpha_{30}\left(F-F_{c}\right) F_{c}^{\prime} \\
w \sim\left(\frac{-\alpha_{11} F_{c}^{\prime}}{\alpha_{30}} z\right)^{\frac{1}{2}}+\left(-2 \sigma^{2} \alpha_{30} F_{c}^{\prime}\right)^{-\frac{1}{4}} \rho_{0}^{*} z^{-\frac{1}{4}} \\
\times \cos \left\{\frac{2 \sqrt{2}}{3}\left(\alpha_{11} F_{c}^{\prime}\right)^{\frac{1}{2}} z^{\frac{3}{2}}-\frac{3 \rho_{0}^{*^{2}}}{4 \sigma^{2} F_{c}^{\prime}} \ln (z)+\psi_{0}^{*}\right\} \\
\quad \text { as } z \rightarrow+\infty
\end{gathered}
$$


with $\rho_{0}, \rho_{0}^{*}, \psi_{0}$, and $\psi_{0}^{*}$ depending on the initial values, on the parameter $\varepsilon$, and on $t_{c}$, the bifurcation moment.

In the region in which $F=O\left(\varepsilon^{2 / 3}\right)$ a new local approximation is constructed, obeying the significant degeneration

$$
\frac{d^{2} y}{d z^{2}}=\alpha_{11} F_{c}^{\prime} z y+\alpha_{30} y^{3}
$$

and its validity is proven in the same way as in $\S 5$. Again, the validity domain can be extended forward and backward using the extension theorem of Eckhaus (1979). Overlap with the outer approximations is ensured so that integration constants follow from matching with these approximations. With the result of Its, Fokas, and Kapaev (1994) the parameters $(\alpha, \varphi)=\left(\left(2 \sigma^{2} F_{c}^{\prime}\right)^{-1 / 2} \rho_{0}, \psi_{0}\right)$ and $(\rho, \theta)=\left(\left(2 \sigma^{2} F_{c}^{\prime}\right) \rho_{0}^{*}, \psi_{0}^{*}\right)$ are connected. In fact, using the transformations

$$
y=i \cdot\left(\frac{-2}{\alpha_{30}}\right)^{\frac{1}{2}}\left(\alpha_{11} F_{c}^{\prime}\right)^{\frac{1}{3}} w, z=\left(\alpha_{11} F_{c}^{\prime}\right)^{-\frac{1}{3}} s
$$

we obtain

$$
\frac{d^{2} w}{d s^{2}}=s w+2 w^{3}
$$

In this way the asymptotic validity of formal expansions of (7.1) is proven. Moreover, if the initial values and the small parameter $\varepsilon$ are known, we can predict the branch approached and the behaviour of the solution after bifurcation. We emphasize that, in contrast with Haberman (1979), the terms

$$
\left.\frac{-1}{4} \rho^{2} \omega^{-1}(F) \frac{\partial^{3} \sigma}{\partial x^{3}}\right|_{x_{s v e}}
$$

and

$$
\frac{-5}{12} \rho^{2} \omega^{-3}(F)\left(\left.\frac{\partial^{2} \sigma}{\partial x^{2}}\right|_{x_{s v e}}\right)^{2}
$$

which appear after averaging over the phase, are of the same order as $F \downarrow F_{c}$ beyond the bifurcation point. Before the bifurcation, we have $\left|5 \omega^{-2}(F)\left(\partial^{2} \sigma /\left.\partial x^{2}\right|_{x s v e}\right)^{2}\right| \ll$ $\left|3 \partial^{3} G / \partial x^{3}\right|_{\text {xsve }} \mid$ for $F \uparrow F_{c}$. This is the reason why we have to apply second-order averaging to approximate the solution beyond the bifurcation point, whereas firstorder averaging is satisfactory for approximation of the solution before this point.

8. Conclusions. In this paper we have analysed a second-order bifurcation system with a slowly varying parameter. We considered the case of a pitchfork bifurcation for which the leading-order transition layer equation is the second Painlevé transcendent. Solutions of this equation either exponentially decay, corresponding to the transition to the unstable slowly varying equilibrium beyond the bifurcation point, or algebraically grow, corresponding to the transition from the equilibrium that turned unstable to one or the other of the two stable branches of the parabolic curve. The transitions are illustrated in Figure 9.

The solution of the problem we formulated is approximated asymptotically using perturbation techniques. The use of both averaging and boundary layer methods turned out to be necessary. The aim of this study was to predict which branch 
(a)

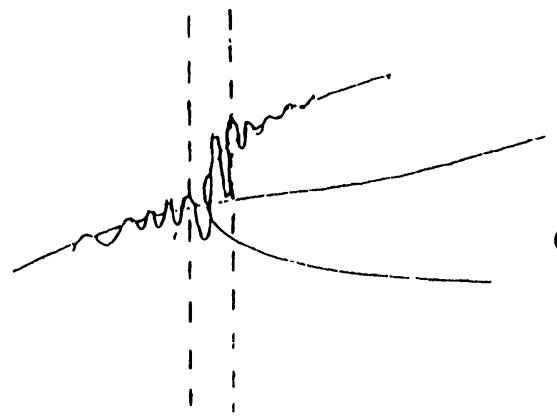

(b)

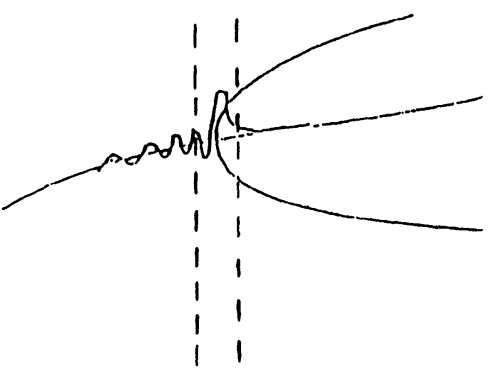

FIG. 9. For a second-order pitchfork bifurcation the second Painleve transcendent provides the transition between two stable slowly varying equilibria (a) or the rare transition to the unstable state (b).

would be followed after passage of the bifurcation point, given the initial state of the system. With the aid of averaging methods the dynamics on the large time-scale are described. Afterward, the validity of the asymptotic approximation is investigated. It is proved that the different local solutions overlap. An analytical study of the transition layer equation produces the required information on the matching of the different locally valid asymptotic approximations. Its and Kapaev (1994) show that there is a connection between the constants of the slowly oscillating solutions that are valid before and after passage of the bifurcation point. Using this result we can predict which stable branch of the parabolic equilibrium curve will be followed by the solution after passage of the bifurcation point, given the initial values "far away" from the bifurcation moment. Moreover, the behaviour of the solution beyond the bifurcation point can again be described by averaging methods. The bifurcation takes place on a relatively small time-scale of length $\varepsilon t=O\left(\varepsilon^{2 / 3}\right)$. In this study the slow increase of the parameter depends only on time. The influence of the state variable on the change of the slowly varying parameter will be a goal for further research.

Acknowledgments. I would like to thank Johan Grasman for his stimulating comments on the investigations which resulted in this paper. I am also grateful to the referees for their useful remarks.

\section{REFERENCES}

S. M. BAer, T. ERneUX, AND J. Rinzel (1989), The slow passage through a Hopf bifurcation: Delay, memory effects and resonance, SIAM J. Appl. Math., 49, pp. 55-71.

D. L. Bosley AND J. KEVORKIAN, Adiabatic invariance and transient resonance in very slowly varying oscillatory Hamiltonian systems, SIAM J. Appl. Math., 52, pp. 494-527.

F. J. Bourland AND R. HABERMAN (1990), Separatrix crossing: Time-invariant potentials with dissipation, SIAM J. Appl. Math., 50, pp. 1716-1744.

(1994), Connection across a separatrix with dissipation, Stud. Appl. Math., 91, pp. 95-124.

F. J. Bourland, R. HABerman, AND W. L. KATH (1991), Averaging methods for the phase shift of arbitrarily perturbed strongly nonlinear oscillators with an application to capture, SIAM J. Appl. Math., 51, pp. 1150-1167.

D. R. J. Chillingworth (1976), Differentiable Topology with a View to Applications, Pitman, London.

P. A. Deift AND X. Zhou (1993), Asymptotics for the Painlevé II Equation: Announcement of results, in Spectral and Scattering Theory and Its Applications, Adv. Stud. Pure Math., Kinokiniya-Shoten, Tokyo, American Mathematical Society. 
J. P. DenieR AND R. GRIMShaw (1990), Slowly-varying bifurcation theory in dissipative systems, J. Austral. Math. Soc. Ser. B, 3, pp. 301-318.

W. Eckhaus (1979), Asymptotic Analysis of Singular Perburbations, North-Holland, Amsterdam, New York, Oxford.

H. FlaschKa AND A. C. NEWEll (1980), Monodromy- and spectrum-preserving deformations I, Comm. Math. Phys., 76, pp. 65-116.

R. HABERMAN (1979), Jump and transition phenomena, SIAM J. Appl. Math., 37, pp. 69-106.

J. HENRARD (1993), The adiabatic invariant in classical mechanics, in Dynamics Reported, Vol. 2, New Series, Springer-Verlag.

L. Holden AND T. ERnEUX (1993), Slow passage through a Hopf bifurcation: From oscillatory to steady state solutions, SIAM J. Appl. Math., 53, pp. 1045-1058.

A. R. Its, A. S. FoKAS, AND A. A. KAPAeV (1994), On the asymptotic analysis of the Painlevé equations via the isomonodromy method, Nonlinearity, 7, pp. 1291-1325.

A. R. ITS AND V. YU. NovoKshenov, The Isomonodromic Deformations Method in the Theory of Painlevé Equations, Lecture Notes in Math. 1191, Springer-Verlag, Berlin, Heidelberg, New York, Tokyo, 1986.

A. V. KITAEV (1989), The justification of the asymptotic formulae obtained by the isomonodromic deformation method, LOMI 179, Mathematical Questions of the Wave Propagation Theory 19, Zap. Nauch. Semin. (In Russian.)

D. LeVi AND P. Winternitz (1992), Painlevé transcendents: Their asymptotics and physical applications, in Proceedings of a NATO Advanced Research Workshop, September 3-7, 1990, Sainte Adele, Quebec, Canada.

G. J. M. MARÉE (1993), Sudden change in a second-order non-linear system with a slowly varying parameter, Internat. J. Non-Linear Mech., 28, pp. 409-426.

A. L. Neishtadt (1986), Change in adiabatic invariant at a separatrix, Sov. J. Plasma Phys., 12, pp. $568-573$.

(1987), Persistence of stability loss for dynamical bifurcations I, Differential Equations, 23, pp. 1385-1391.

(1988), Persistence of stability loss for dynamical bifurcations II, Differential Equations, 24, pp. 171-176.

(1993), On probabilistic phenomena in perturbed systems, Selecta Mechanica, 12, pp. 195-201.

PAINlevé (1900), Etude de l'equation $y^{\prime \prime}=6 y^{2}+x$, Bull. Soc. Math. France, 28, pp. 227-252.

J. Sanders and F. Verhulst (1985), Averaging Methods in Nonlinear Dynamical Systems, Appl. Math. Sci. 59, Springer-Verlag, New York.

J. J. STOKER (1950), Nonlinear Vibrations in Mechanical and Electrical Systems, Interscience, New York.

F. Verhulst (1990a), Basic perturbation theory and asymptotic methods, Lecture notes, State University Utrecht.

(1990), Nonlinear Differential Equations and Dynamical Systems, Springer-Verlag, Berlin.

V. E. ZAKhaROV (1991), What Is Integrability?, Springer Ser. Nonlinear Dynamics, Springer-Verlag, New York. 